\title{
Steady-state solutions in a nonlinear pool boiling model
}

\author{
Michel Speetjens, Arnold Reusken*† Wolfgang Marquardt ${ }^{\ddagger}$ \\ RWTH Aachen, Templergraben 55, D-52056 Aachen, Germany.
}

\begin{abstract}
We consider a relatively simple model for pool boiling processes. This model involves only the temperature distribution within the heater and describes the heat exchange with the boiling fluid via a nonlinear boundary condition imposed on the fluid-heater interface. This results in a standard heat equation with a nonlinear Neumann boundary condition on part of the boundary. In this paper we analyse the qualitative structure of steady-state solutions of this heat equation. It turns out that the model allows both multiple homogeneous and multiple heterogeneous solutions in certain regimes of the parameter space. The latter solutions originate from bifurcations on a certain branch of homogeneous solutions. We present a bifurcation analysis that reveals the multiple-solution structure in this mathematical model. In the numerical analysis a continuation algorithm is combined with the method of separation-of-variables and a Fourier collocation technique. For both the continuous and discrete problem a fundamental symmetry property is derived that implies multiplicity of heterogeneous solutions. Numerical simulations of this model problem predict phenomena that are consistent with laboratory observations for pool boiling processes.
\end{abstract}

PACS 44.35.+c, 44.05.+e, 02.60.Cb, 02.70.Jn

Keywords. pool boiling, nonlinear heat transfer, bifurcation analysis, numerical simulation

\section{Introduction}

Pool boiling refers to boiling processes that lean on natural convection as a means for heat transfer between a heater surface and the boiling fluid; it is the key mode of thermal transport in many practical applications. Local heat transfer phenomena near heating walls in industrial boiling equipment (e.g. evaporators and kettle reboilers) for instance are essentially pool boiling processes [1]. Furthermore, pool boiling is emerging as novel cooling technique for electronics components [2]. Despite its importance, many aspects of (pool) boiling remain largely unexplored to date, mainly due to the immense complexity of the process emanating from the intricate interplay between fluid dynamics, heat transfer from the heater to the fluid and phase transfer. Studies on boiling known in the literature are mainly experimental and empirical. Numerical studies based on a model of reasonable degree of detail are numerous but theoretical investigations aiming at a rigorous analysis of such models are scarce. The

\footnotetext{
${ }^{*}$ Chair of Numerical Analysis. This author was supported by the German Research Foundation through the Research Training Group "Hierarchy and Symmetry in Mathematical Models"

${ }^{\dagger}$ Corresponding author: Chair of Numerical Analysis, RWTH Aachen, Templergraben 55, D-52056 Aachen, Germany, +49-241-80-97973, reusken@igpm.rwth-aachen.de

${ }^{\ddagger}$ Chair of Process Systems Engineering
} 
theoretical analysis of a simple boiling model presented in this paper is intended to contribute to a better qualitative understanding of fundamental phenomena in pool boiling.

In pool boiling there are three fundamental boiling modes, i.e. nucleate, transition and film boiling, that occur successively with increasing temperature [3]. Nucleate boiling is, as opposed to film boiling, an efficient and safe mode of heat transfer and is the desired boiling mode in most practical applications. Nucleate boiling transits into film boiling upon exceeding the so-called critical heat flux (CHF) through the intermediate state of transition boiling. This transition results in a dramatic increase in interface temperature due to the substantial drop in the heat-transfer coefficient when going from nucleate boiling (homogeneous liquidlike mixture) to film boiling (vapour blanket on the interface). This manifests itself in the essentially nonlinear relation between the mean heat flux and the mean interface temperature (the so-called boiling curve [3]). Improving the efficiency of boiling processes involves finding a good balance between high heat-transfer coefficients (close to CHF) and low risk (safe distance from CHF). In-depth understanding of transition boiling and its underlying mechanisms is imperative to achieving such a balance [4].

Transition boiling may in a simplified description be considered as a state of "two-mode boiling" that consists of coexisting nucleate-boiling and film-boiling regions [5]. A more intricate and most likely more precise description of the two-phase structure in transition boiling has been derived in a series of papers by Auracher and co-workers (see [6] for a survey). Moreover, transition boiling is an inherently unstable state that naturally evolves towards one of the two stable boiling modes, i.e. nucleate and film boiling, unless actively stabilised through temperature control [7]. On mesoscopic length and time scales two-mode boiling states correspond to heterogeneous temperature fields on the interface: "lower" temperatures correspond to nucleate boiling regions; "higher" temperatures are associated with film boiling regions. ${ }^{1}$ Furthermore, the propagation of boundaries between adjacent boiling regions during evolution of the transition mode towards one of the stable modes is consistent with the propagation of thermal waves at the fluid-heater interface $[9,10]$. This phenomenological connection between a (mesoscopic) boiling mode and interface temperature admits a heateronly modelling approach that omits the boiling fluid and describes the (qualitative) behaviour of the boiling system entirely in terms of the temperature distribution within the heater. The heat exchange between the heater and the fluid is modelled by a nonlinear heat-flux relation that is similar to the boiling curve [10]. The simplification from the multi-phase problem to a heater-only model naturally disqualifies this approach for detailed quantitative studies. However, the heater-only approach allows the analysis of fundamental (mesoscopic) boiling phenomena using numerical analysis. Moreover, the present model serves as point of departure for more sophisticated future models that enable quantitative studies.

The heater-only approach has found widespread application for the analysis of pool boiling on "thin" heaters such as wires and foils [9, 11-15]. In such configurations the heat-flux relation results in a nonlinear source term in the heat governing equations that thus these are very similar to reaction-diffusion systems [10] and generic current-carrying systems [16]. First extensions to finite-thickness heaters, where the heat-flux relation leads to a nonlinear boundary condition and thus an essentially different model, are presented in [10].

The transition behaviour of the heater-only problem basically involves two issues: (i) formation and (ii) dynamics of heterogeneous temperature fields [6]. These two issues lead to

\footnotetext{
${ }^{1}$ Here mesoscopic means locally averaged in space and time over intervals larger than bubble dimensions and bubble lifetimes in order to smooth out microscopic short-term fluctuations [8].
} 
questions concerning existence and stability of steady-state solutions. Analysis of thin heaters has shown the existence of multiple steady-state solutions for given heating conditions. Each set of steady-state solutions appears to accommodate at most two stable solutions; other solutions are unstable [15]. Results obtained for cylindrical heaters with linearised heat-flux relations are consistent with these results [17]. However, similar studies on thick heaters for the generic case of nonlinear heat-flux relations and heterogeneous interface temperatures are restricted to the analysis of [10]. The latter contains a numerical study of the evolution of twomode boiling states towards one of the two stable boiling modes (i.e nucleate or film boiling) on a $2 \mathrm{D}$ thick heater. Initial two-mode boiling states (unstable steady states) are represented by discontinuous - and thus rather artificial - temperature profiles, though. Numerical studies on realistic heterogeneous steady-state solutions are not known to the authors.

In this paper we present an extensive analysis of the steady-state behaviour of a spatially two-dimensional (2D) thick heater problem. We use the heat-transfer model proposed in [18], based upon that introduced by [10], and show that multiple steady-state solutions may occur in this model for specific conditions. Central topic is the dependence of the multiple solution structure on the system parameters. To this end a bifurcation analysis is performed on the model in order to identify solution branches and bifurcations as a function of the system parameters. This analysis is based on a numerical continuation algorithm combined with the method of separation-of-variables and a Fourier collocation method.

The paper is organised as follows. Section 2 introduces the model problem. Key properties of this model are derived in Section 3. Section 4 elaborates on the discretisation method and the continuation algorithm. Section 5 demonstrates this methodology with a representative case study. Section 6 presents a generic bifurcation analysis of the model problem. Conclusions are in Section 7.

\section{Problem definition and mathematical model}

Our pool boiling investigations are based on the heater-only modelling approach introduced in Section 1 and following [10]. We consider the two-dimensional rectangular heater $\mathcal{D}=$ $[0, L] \times[0, H]$, with boundary $\Gamma=\partial \mathcal{D}=\Gamma_{H} \cup \Gamma_{A} \cup \Gamma_{F}$. The boundary segments are $\Gamma_{H}=$ $\{(x, y) \in \mathcal{D} \mid y=0\}$ (heat supply), $\Gamma_{A}=\{(x, y) \in \mathcal{D} \mid x=0$ or $x=L\}$ (adiabatic sidewalls) and $\Gamma_{F}=\{(x, y) \in \mathcal{D} \mid y=H\}$ (fluid-heater interface). The heat transfer is described in terms of the superheat $T$, i.e. the temperature excess beyond the boiling point. The steady-state temperature distribution $T(\boldsymbol{x})$ in $\mathcal{D}$ is governed by the heat equation

$$
\Delta T=0,\left.\quad \lambda \frac{\partial T}{\partial n}\right|_{\Gamma_{H}}=\bar{q}_{H}, \quad-\left.\lambda \frac{\partial T}{\partial n}\right|_{\Gamma_{F}}=\bar{q}_{F}\left(T_{F}\right),\left.\quad \frac{\partial T}{\partial n}\right|_{\Gamma_{A}}=0,
$$

where $T_{F}$ denotes the interface temperature on the boundary segment $\Gamma_{F}$ and $n$ the outward pointing normal. The constant $\lambda$ is the thermal conductivity of the heater; $\bar{q}_{H}$ and $\bar{q}_{F}$ represent the constant heat supply and temperature-dependent heat transfer to the boiling fluid, respectively.

Closure of the heat-transfer model requires specification of the heat-flux function $\bar{q}_{F}\left(T_{F}\right)$. Physical considerations suggest local heat-transfer coefficients specific to liquid and vapour contact for "lower" and "higher" local interface temperatures $T_{F}$, respectively. This implies that the function $\bar{q}_{F}\left(T_{F}\right)$ should be qualitatively similar to the global boiling curve. For simplicity, we identify $\bar{q}_{F}\left(T_{F}\right)$ with the functional form of the global boiling curve (Figure $1 a$ ). This boiling curve consist of three distinct regimes that each correspond to one of the boiling 
modes: nucleate boiling $\left(0 \leq T \leq T_{C}\right)$; transition boiling $\left(T_{C}<T<T_{M}\right)$; film boiling $\left(T \geq T_{M}\right)$. The nucleate and film boiling regions represent local liquid and vapour contacts respectively; the transition region is modeled by a smooth connection between the different heat-transfer coefficients of adjacent liquid and vapour contacts. An explicit expression for $\bar{q}_{F}\left(T_{F}\right)$ is given below.

We formulate the heat-transfer problem (1) in non-dimensional form through rescaling of the variables: $\boldsymbol{x}^{\prime}=\boldsymbol{x} / L, T^{\prime}=T / T_{D}, q_{H}^{\prime}=\bar{q}_{H} / Q_{H}$ and $q_{F}^{\prime}=\bar{q}_{F} / Q_{C}$. Substitution into the governing equations and dropping primes yields the non-dimensional model

$$
\Delta T=0,\left.\quad \Lambda \frac{\partial T}{\partial n}\right|_{\Gamma_{H}}=1, \quad-\left.\Lambda \frac{\partial T}{\partial n}\right|_{\Gamma_{F}}=\Pi_{2} q_{F}\left(T_{F}\right),\left.\quad \frac{\partial T}{\partial n}\right|_{\Gamma_{A}}=0
$$

on the non-dimensional rectangular heater $\mathcal{D}=[0,1] \times[0, D]$, with system parameters

$$
\Lambda=\frac{\lambda T_{D}}{Q_{H} L}, \quad D=\frac{H}{L}, \quad \Pi_{1}=\frac{Q_{C}}{Q_{M}}, \quad \Pi_{2}=\frac{Q_{C}}{Q_{H}}, \quad \Pi_{3}=\frac{T_{C}}{T_{M}} .
$$

Here $Q_{H}$ is a fixed typical value for the heat supply; in the current case of a constant heat supply we use $Q_{H}=\bar{q}_{H}$ (i.e. $q_{H}^{\prime}=1$ ). Note that $q_{F}=\bar{q}_{F} / Q_{C}$ is the normalised boiling curve (i.e. rescaled with $Q_{C}$ instead of $Q_{H}$ ).

The normalised boiling curve can be parameterised by

$$
q_{F}\left(T_{F}\right)=h\left(T_{F}\right) T_{F}, \quad h\left(T_{F}\right):=C_{D}\left\{F_{1}-F_{2} H\left(C_{D} T_{F}-1\right)\right\},
$$

with $h\left(T_{F}\right)$ the temperature-dependent heat-transfer coefficient and $H(\zeta)=\frac{1}{2}\left[\tanh \left(\frac{2 \zeta}{W}\right)+1\right]$ a smoothed version of the Heaviside function. The parameter $W$ sets the width of the transition region (from $H=0$ to $H=1$ ) around $\zeta=0$ and is specified a-priori. The coefficient $C_{D}$ rescales the temperature such that the single deflection point of $q_{F}\left(T_{F}\right)$ coincides with $T_{F}=1$. Its value is defined implicitly through $2 \frac{d H}{d T}\left(C_{D}-1\right)+\frac{d^{2} H}{d T^{2}}\left(C_{D}-1\right)=0$ and depends only on $W$. The coefficients $F_{1}$ and $F_{2}$ scale $q_{F}\left(T_{F}\right)$ such that the four conditions $\dot{q}_{F}\left(T_{\max }\right)=0, \dot{q}_{F}\left(T_{\min }\right)=0, q_{F}\left(T_{\max }\right)=1$ and $q_{F}\left(T_{\min }\right)=\Pi_{1}^{-1}$ are fulfilled, i.e. the extrema of the normalised boiling curve are consistent with their dimensional counterparts, where $\dot{q}_{F}=d q_{F} / d T$. These conditions determine $\left(F_{1}, F_{2}, T_{\min }, T_{\max }\right)$, with $T_{\max }<1$ (local maximum) and $T_{\text {min }}>1$ (local minimum) for given $W$ and $\Pi_{1}$. Figure $1 b$ shows $q_{F}$ for $W=1$ and $\Pi_{1}=4$. Note that $W$ indirectly sets $\Pi_{3}$; both parameters may therefore be used interchangeably without loss of generality. The present boiling curve is a generalisation of the one proposed in [10] in the sense that here the transition width $W$ is an additional system parameter.

\section{Analysis of the steady-state problem}

The method of separation-of-variables [19] enables derivation of a (formal) solution of the Laplace equation and the linear Neumann conditions on $\Gamma_{H}$ and $\Gamma_{A}$ in (2). This results in

$$
T(x, y)=\sum_{n=0}^{\infty} \widetilde{T}_{n} \frac{\cosh (n \pi y)}{\cosh (n \pi D)} \cos (n \pi x)+\frac{D-y}{\Lambda},
$$

where the coefficients $\widetilde{T}_{n}$ form the spectrum of the Fourier cosine expansion

$$
T_{F}(x):=T(x, D)=\sum_{n=0}^{\infty} \widetilde{T}_{n} \cos (n \pi x)
$$




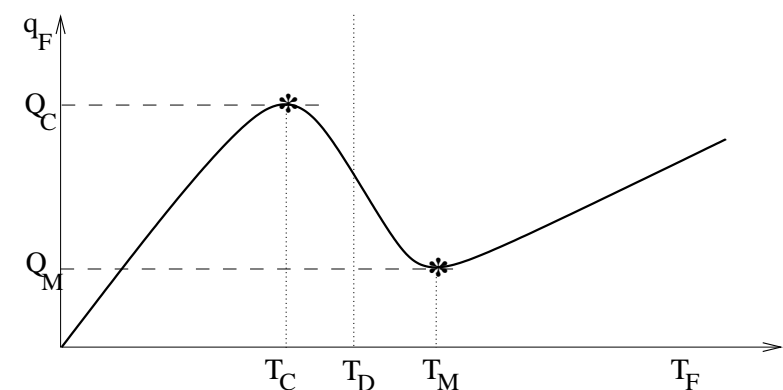

a) Global boiling curve.

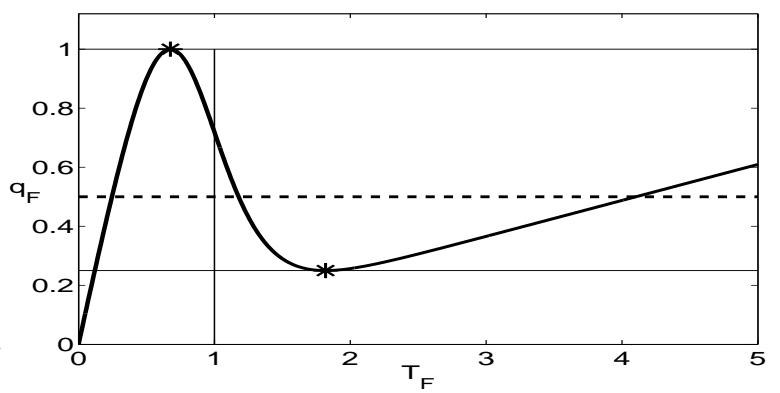

b) Normalised boiling curve $q_{F}$.

Figure 1: Definition of heat-flux function $q_{F}$. Panel a gives the global boiling curve. Temperatures $T_{C}$ and $T_{M}$ coincide with the extrema (stars); $T_{D}$ is a typical temperature during transition boiling. Panel $b$ gives the corresponding normalised boiling curve $q_{F}$ for $\Pi_{1}=4$ and $W=1 . T=1$ is the non-dimensional counterpart of $T_{D}$; the extrema (stars) correspond with $q_{F}=1$ and $q_{F}=\Pi_{1}^{-1}$. The dashed line represents the normalised heat supply $\bar{q}_{H} / Q_{C}=\Pi_{2}^{-1}$.

of the interface temperature. The spectrum $\widetilde{T}_{n}$ is determined by the nonlinear Neumann condition on $\Gamma_{F}$. Substitution of (5) into this nonlinear condition and using (4) leads to

$$
\sum_{n=0}^{\infty} n \pi \tanh (n \pi D) \widetilde{T}_{n} \cos (n \pi x)+\alpha\left(T_{F}(x)\right) T_{F}(x)-\frac{1}{\Lambda}=0 \text { for all } x \in[0,1],
$$

where

$$
\alpha\left(T_{F}\right)=\frac{\Pi_{2}}{\Lambda} h\left(T_{F}\right)=\frac{\Pi_{2}}{\Lambda} \frac{q_{F}\left(T_{F}\right)}{T_{F}},
$$

is the scaled heat-transfer coefficient. The nonlinear equation (7) determines the solution(s) of (2). Thus the $2 D$ problem (2) simplifies to the $1 D$ problem (7) involving only the temperature profile $T_{F}(x)$ on the boundary $\Gamma_{F}$. The series in (6) and (7) are formal expressions; their convergence is discussed below.

We first consider the special case of spatially homogeneous interface temperatures $T_{F}$, implying $T_{F}(x)=\widetilde{T}_{0}$ and $\widetilde{T}_{n}=0$ for $n>0$. Then the nonlinear condition (7) simplifies to

$$
q_{F}\left(\widetilde{T}_{0}\right)=\Pi_{2}^{-1},
$$

and $\widetilde{T}_{0}$ coincide(s) with the intersection(s) between the boiling curve (solid line in Figure $1 b$ ) and the normalised heat supply $\bar{q}_{H} / Q_{C}=\Pi_{2}^{-1}$ (dashed line in Figure $1 b$ ). From Figure $1 b$ it follows that, depending on the system parameters, we can have one, two or three solutions

for $\widetilde{T}_{0}$. Note that in this homogeneous case the heat-transfer coefficient $h\left(T_{F}\right)$ is constant and thus the Neumann boundary condition on $\Gamma_{F}$ is linear. The corresponding solution in $\mathcal{D}$ is given by

$$
T(x, y)=\frac{D}{\Lambda}\left(1-\frac{y}{D}\right)+\widetilde{T}_{0},
$$

defining a linear profile with constant interface temperature $T_{F}(x)=\widetilde{T}_{0}$ given by $(8)$.

We now return to the general case and derive two properties important for the analysis below. We introduce the Fourier space of convergent Fourier cosine series

$$
V_{k}:=\left\{g: \mathbb{R} \rightarrow \mathbb{R} \mid g(x)=\sum_{n=0}^{\infty} a_{k n} \cos (k n \pi x) \quad \forall x \in \mathbb{R}\right\}, \quad k=1,2, \ldots
$$


Functions from this space are $\frac{2}{k}$-periodic and even (i.e. $\left.g(x)=g(-x)\right)$ and are uniquely determined by their values at $x \in\left[0, \frac{1}{k}\right]$. For the Fourier coefficients $a_{k n}$ we have the representation

$$
a_{0}=\int_{0}^{1} g(x) d x, \quad a_{k n}=2 \int_{0}^{1} g(x) \cos (k n \pi x) d x \quad(k n>0) .
$$

Note that $V_{2 k} \subset V_{k}$ for all $k$. The Fourier transform on $V_{1}$ is denoted by $\mathcal{F}: V_{1} \rightarrow \ell^{2}$ :

$$
\text { for } g(x)=\sum_{n=0}^{\infty} a_{n} \cos (n \pi x), \quad \mathcal{F}(g):=\left(a_{n}\right)_{n \geq 0} .
$$

For $\left(b_{n}\right)_{n \geq 0},\left(c_{n}\right)_{n \geq 0} \in \ell^{2}$ we define the product $\left(b_{n}\right)_{n \geq 0} \cdot\left(c_{n}\right)_{n \geq 0}:=\left(b_{n} c_{n}\right)_{n \geq 0}$, i.e., elementwise multiplication of the entries in the sequences. Furthermore, we define the sequence

$$
\mathbf{d}=\left(d_{n}\right)_{n \geq 0}, \quad d_{n}:=n \pi \tanh (n \pi D) \text { for all } n .
$$

To guarantee that the expressions on the left handside in (7) are well-defined we only consider functions from the following subset of $V_{1}$ :

$$
S:=\left\{g \in V_{1} \mid \mathbf{d} \cdot \mathcal{F}(g) \in \operatorname{range}(\mathcal{F}) \text { and }(\alpha \circ g) g \in V_{1}\right\}
$$

Remark 1 Functions $g \in V_{1}$ that are sufficiently smooth are elements of $S$. We do not study this issue here, but only give one simple result related to this. Elementary Fourier analysis yields that if $g \in V_{1}$ and $g \in C^{3}(\mathbb{R})$ then $g \in S$ holds.

The operator on the left handside in (7) has the following form

$$
\mathcal{G}\left(T_{F}\right):=\mathcal{F}^{-1}\left(\mathbf{d} \cdot \mathcal{F}\left(T_{F}\right)\right)+\left(\alpha \circ T_{F}\right) T_{F}-\frac{1}{\Lambda}, \quad \text { for } \quad T_{F} \in S .
$$

The definition of $S$ implies $\mathcal{G}: S \rightarrow V_{1}$. Thus (7) leads to the following problem:

$$
\text { Determine } T_{F} \in S \text {, such that } \mathcal{G}\left(T_{F}\right)=0 \text {. }
$$

The operator $\mathcal{G}$, defined on $S$, is (strongly) nonlinear. The homogeneous solutions given by (8) satisfy $\mathcal{G}\left(\widetilde{T}_{0}\right)=0$. We now show that for every $k \geq 1$ the range of $\mathcal{G}_{\mid V_{k}}$ is contained in $V_{k}$.

Theorem 1 The following holds:

$$
\mathcal{G}: V_{k} \cap S \rightarrow V_{k} \quad \text { for all } k \geq 1 \text {. }
$$

Proof. For $k=1$ this is trivial due to the definition of $S$. Solutions $T_{F} \in V_{k} \cap S$ can be represented as $T_{F}(x)=\sum_{n=0}^{\infty} \widetilde{T}_{k n} \cos (k n \pi x)$, and $\widetilde{T}_{m}=0$ for $m \bmod k \neq 0$. We obtain

$$
\begin{aligned}
\mathcal{G}\left(T_{F}\right)(x) & =\sum_{n=0}^{\infty} d_{n} \widetilde{T}_{n} \cos (n \pi x)+\alpha\left(T_{F}(x)\right) T_{F}(x)-\frac{1}{\Lambda} \\
& =\sum_{n=0}^{\infty} d_{k n} \widetilde{T}_{k n} \cos (k n \pi x)+\alpha\left(T_{F}(x)\right) T_{F}(x)-\frac{1}{\Lambda} \\
& =: w_{1}(x)+w_{2}(x)-\frac{1}{\Lambda} .
\end{aligned}
$$


From $T_{F} \in S$ it follows that the series $w_{1}(x)=\sum_{n=0}^{\infty} d_{k n} \widetilde{T}_{k n} \cos (k n \pi x)$ converges and thus $w_{1} \in V_{k}$. From $T_{F} \in S$ it also follows that $w_{2}=\left(\alpha \circ T_{F}\right) T_{F} \in V_{1}$ and thus $w_{2}$ has a convergent cosine Fourier series. Furthermore, because $T_{F}$ is $\frac{2}{k}$-periodic and even it follows that $w_{2}$ is $\frac{2}{k}$-periodic and even, and thus $w_{2} \in V_{k}$. Hence, we have $\mathcal{G}\left(T_{F}\right)=w_{1}+w_{2}-\frac{1}{\Lambda} \in V_{k}$.

In the next theorem we present a symmetry property of heterogeneous solutions.

Theorem 2 Assume that there exists $k \geq 1$ and $T_{F} \in V_{k} \cap S$ such that $T_{F} \notin V_{\ell}$ for $\ell>k$ and

$$
T_{F}(x)=\sum_{n=0}^{\infty} \widetilde{T}_{k n} \cos (k n \pi x)
$$

satisfies $\mathcal{G}\left(T_{F}\right)=0$. Define

$$
T_{F}^{*}(x):=T_{F}\left(x+\frac{1}{k}\right)=\sum_{n=0}^{\infty} \widetilde{T}_{k n}^{*} \cos (k n \pi x), \quad \text { with } \widetilde{T}_{k n}^{*}=(-1)^{n} \widetilde{T}_{k n} .
$$

Then $T_{F}^{*} \in V_{k} \cap S$ satisfies $\mathcal{G}\left(T_{F}^{*}\right)=0$, and $T_{F}^{*} \neq T_{F}$.

Proof. Since $T_{F} \in V_{k} \cap S$ is even and $\frac{2}{k}$-periodic and $T_{F}^{*}$ is a translation of $T_{F}$ by $\frac{1}{k}$ it follows that $T_{F}^{*} \in V_{k} \cap S$. Using

$$
T_{F}\left(x+\frac{1}{k}\right)=\sum_{n=0}^{\infty} \widetilde{T}_{k n} \cos \left(k n \pi\left(x+\frac{1}{k}\right)\right)=\sum_{n=0}^{\infty}(-1)^{n} \widetilde{T}_{k n} \cos (k n \pi x)
$$

we obtain the representation in (14). Note that

$$
T_{F}(x)-T_{F}^{*}(x)=\sum_{n=0}^{\infty}\left(1-(-1)^{n}\right) \widetilde{T}_{k n} \cos (k n \pi x) .
$$

Assume that $T_{F}=T_{F}^{*}$ holds. Then $\widetilde{T}_{k n}=0$ must hold for all odd $n$, and thus we obtain the representation $T_{F}(x)=\sum_{n=0}^{\infty} \widetilde{T}_{2 k n} \cos (2 k n \pi x)$. This implies $T_{F} \in V_{2 k}$, which contradicts the assumption $T_{F} \notin V_{\ell}$ for $\ell>k$. Thus $T_{F}^{*} \neq T_{F}$ must hold. For arbitrary $x \in \mathbb{R}$ we have

$$
\begin{aligned}
\mathcal{G}\left(T_{F}^{*}\right)(x) & =\mathcal{F}^{-1}\left(\mathbf{d} \cdot \mathcal{F}\left(T_{F}^{*}\right)\right)(x)+\alpha\left(T_{F}^{*}(x)\right) T_{F}^{*}(x) \\
& =\sum_{n=0}^{\infty} d_{k n}(-1)^{n} \widetilde{T}_{k n} \cos (k n \pi x)+\alpha\left(T_{F}\left(x+\frac{1}{k}\right)\right) T_{F}\left(x+\frac{1}{k}\right) \\
& =\sum_{n=0}^{\infty} d_{k n} \widetilde{T}_{k n} \cos \left(k n \pi\left(x+\frac{1}{k}\right)\right)+\alpha\left(T_{F}\left(x+\frac{1}{k}\right)\right) T_{F}\left(x+\frac{1}{k}\right) \\
& =\mathcal{F}^{-1}\left(\mathbf{d} \cdot \mathcal{F}\left(T_{F}\right)\right)\left(x+\frac{1}{k}\right)+\alpha\left(T_{F}\left(x+\frac{1}{k}\right)\right) T_{F}\left(x+\frac{1}{k}\right) \\
& =\mathcal{G}\left(T_{F}\right)\left(x+\frac{1}{k}\right)=0 .
\end{aligned}
$$

Hence, $\mathcal{G}\left(T_{F}^{*}\right)=0$ holds.

This result shows that heterogeneous solutions in $V_{1} \cap S$, if they exist, always occur as a 
conjugate pair: (13) and (14). (Note that the dual solution $T_{F}^{*}$ is obtained from $T_{F}$ by a translation with half the period of $T_{F}$.) This implies a fundamental non-uniqueness in the steady states under heterogeneous boiling conditions, consistent with laboratory experiments [6].

In the proof of Theorem 2 we derived the following fundamental property of the operator $\mathcal{G}$. Let $s_{k}: \mathbb{R} \rightarrow \mathbb{R}$ be the linear shift function $s_{k}(x):=x+\frac{1}{k}$. For $T_{F} \in V_{k} \cap S$ the relation

$$
\mathcal{G}\left(T_{F} \circ s_{k}\right)=s_{k} \circ \mathcal{G}\left(T_{F}\right)
$$

holds. Due to this commutator property of the nonlinear operator $\mathcal{G}$ and the linear shift operator $s_{k}$ we obtain the symmetry result in Theorem 2 .

Remark 2 The form of the function $\alpha(\cdot)$ is immaterial for the proofs of Theorem 1 and Theorem 2. Hence these results hold for an arbitrary (smooth) boiling curves $q_{F}$.

\section{Numerical solution method}

The steady-state solutions follow from the characteristic equation (7). For homogeneous solutions the latter simplifies to (8) and can be resolved by a standard root-finding algorithm. Thus homogeneous solution branches are readily identified. Heterogeneous solutions, on the other hand, have to be determined via the discretisation and continuation approach elaborated hereafter. Numerical results obtained with this method will be presented in Sections 5 and 6 .

\subsection{Discretisation method}

Discretisation of (7) is based on a standard Fourier collocation method [20]. We briefly review a few basic facts from discrete Fourier analysis. Consider for $N \in \mathbb{N}$ the equidistant mesh $x_{j}=j / N, j \in \mathbb{N}$. The discrete Fourier cosine transform of an even 2-periodic function $u(x)=u(x+2)$ is given by

$$
\sum_{n=0}^{N} \widetilde{u}_{n} \cos (n \pi x), \quad \widetilde{u}_{n}:=\frac{c_{n}}{N}\left\{u(0)+2 \sum_{j=1}^{N-1} u\left(x_{j}\right) \cos \left(n \pi x_{j}\right)+(-1)^{n} u(1)\right\},
$$

with $c_{0}=c_{N}=1 / 2$ and $c_{n}=1$ otherwise. This function satisfies $\sum_{n=0}^{N} \widetilde{u}_{n} \cos \left(n \pi x_{i}\right)=u\left(x_{i}\right)$ for all $0 \leq i \leq N$. Hence, the (physical) values $\boldsymbol{u}=\left(u_{0}, \ldots, u_{N}\right)^{T}$, with $u_{j}:=u\left(x_{j}\right)$, relate to the spectral coefficients $\widetilde{\boldsymbol{u}}=\left(\widetilde{u}_{0}, \ldots, \widetilde{u}_{N}\right)^{T}$ via

$$
\boldsymbol{u}=\boldsymbol{V} \widetilde{\boldsymbol{u}}, \quad \boldsymbol{V}=\left(V_{i j}\right)_{0 \leq i, j \leq N}, \quad V_{i j}:=\cos \left(j \pi x_{i}\right) .
$$

An elementary computation yields

$$
(\boldsymbol{V} \boldsymbol{D})^{-1}=\frac{2}{N} \boldsymbol{V} \boldsymbol{D}, \quad \text { with } \boldsymbol{D}=\operatorname{diag}(1 / 2,1, \ldots, 1,1 / 2) .
$$

The discretisation of (7) is as follows: determine $T_{F}\left(x_{j}\right)=\sum_{n=0}^{N} \widetilde{T}_{n} \cos \left(n \pi x_{j}\right), 0 \leq j \leq N$, such that

$$
\sum_{n=0}^{N} d_{n} \widetilde{T}_{n} \cos \left(n \pi x_{j}\right)+\alpha\left(T_{F}\left(x_{j}\right)\right) T_{F}\left(x_{j}\right)-\frac{1}{\Lambda}=0 \quad \text { for all } 0 \leq j \leq N .
$$


This set of $N+1$ (nonlinear) equations for the unknowns $\widetilde{T}_{n}(0 \leq n \leq N)$ or, equivalently, $T_{F}\left(x_{j}\right)(0 \leq j \leq N)$, can be represented in a compact matrix-vector formulation. We use the notation $T_{j}:=T_{F}\left(x_{j}\right)(j=0, \ldots, N), \boldsymbol{t}=\left(T_{0}, \ldots, T_{N}\right)^{T}$ and $\tilde{\boldsymbol{t}}=\left(\widetilde{T}_{0}, \ldots, \widetilde{T}_{N}\right)^{T}$. Note that $\boldsymbol{t}$ consists of the discrete temperature values on the boundary $\Gamma_{F}$ and $\tilde{\boldsymbol{t}}$ contains the corresponding Fourier coefficients. We introduce the diagonal matrices

$$
\boldsymbol{K}_{S}=\operatorname{diag}\left(d_{n}\right)_{0 \leq n \leq N}, \quad \boldsymbol{M}(\boldsymbol{t})=\operatorname{diag}\left(\alpha\left(T_{j}\right)\right)_{0 \leq j \leq N},
$$

the vector $\boldsymbol{g}=(1 / \Lambda, \ldots, 1 / \Lambda)^{T}$ and the matrix $\boldsymbol{K}:=\boldsymbol{V} \boldsymbol{K}_{S} \boldsymbol{V}^{-1}$. Then the discrete problem (19) can be formulated as follows: determine $\boldsymbol{t} \in \mathbb{R}^{N+1}$ such that

$$
\mathcal{G}(\boldsymbol{t}):=(\boldsymbol{K}+\boldsymbol{M}(\boldsymbol{t})) \boldsymbol{t}-\boldsymbol{g}=0 .
$$

The above defines a nonlinear system in the (physical) unknown $\boldsymbol{t}$. The equivalent representation in the (spectral) unknown $\tilde{\boldsymbol{t}}$ is given by

$$
\left(\boldsymbol{K}_{S}+\boldsymbol{M}_{S}(\tilde{\boldsymbol{t}})\right) \tilde{\boldsymbol{t}}=\boldsymbol{V}^{-1} \boldsymbol{g}, \quad \boldsymbol{M}_{S}(\tilde{\boldsymbol{t}}):=\boldsymbol{V}^{-1} \boldsymbol{M}(\boldsymbol{V} \tilde{\boldsymbol{t}}) \boldsymbol{V} .
$$

The nonlinearity of the problems (20) and (21) is contained in the diagonal matrix $\boldsymbol{M}(\boldsymbol{t})$ and the full matrix $\boldsymbol{M}_{S}(\tilde{\boldsymbol{t}})$, respectively. The former admits a more efficient numerical treatment and thus we have used the physical representation (20) in our numerical simulations.

For the discrete nonlinear operator $\mathcal{G}: \mathbb{R}^{N+1} \rightarrow \mathbb{R}^{N+1}$ in (20) we will derive properties similar to those for the continuous operator $\mathcal{G}$ in the Theorems 1 and 2. For this we first introduce some further notation. Let $\mathbf{v}_{m}$ be the $m$-th column of the matrix $\mathbf{V}$ in (17). Define

$$
V_{k}^{N}:=\operatorname{span}\left\{\mathbf{v}_{k n} \mid 0 \leq n \leq \frac{N}{k}\right\}, \quad k=1, \ldots, N .
$$

This space is the discrete analogon of $V_{k}$ in (10). Note that $V_{k}^{N} \subset V_{1}^{N}=\mathbb{R}^{N+1}$ for all $k$. We now give a discrete analogon of Theorem 1 .

Theorem 3 Let $1 \leq k \leq N$ be such that $N \bmod k=0$. The following holds:

$$
\mathcal{G}: V_{k}^{N} \rightarrow V_{k}^{N} \text {. }
$$

The symmetry property for the continuous problem as formulated in Theorem 2 is inherited by the discretisation. We formulate a discrete analogon of Theorem 2 :

Theorem 4 Let $1 \leq k \leq N$ be such that $N \bmod k=0$ and define $m:=\frac{N}{k}$. Assume that there exists $\boldsymbol{t}=\sum_{n=0}^{m} \tilde{t}_{k n} \boldsymbol{v}_{k n} \in V_{k}^{N}$ such that $\boldsymbol{t} \notin V_{\ell}^{N}$ for $\ell>k$ and $\mathcal{G}(\boldsymbol{t})=0$. Define

$$
\boldsymbol{t}^{*}=\sum_{n=0}^{m}(-1)^{n} \tilde{t}_{k n} \boldsymbol{v}_{k n} \text {. }
$$

Then $\boldsymbol{t}^{*} \in V_{k}^{N}$ satisfies $\mathcal{G}\left(\boldsymbol{t}^{*}\right)=0$, and $\boldsymbol{t}^{*} \neq \boldsymbol{t}$.

Proofs of these two theorems require some technical modifications of the proofs of analogous results in the continuous case given in Section 3. These proofs are given in the Appendix. The result in Theorem 4 shows that if a discrete heterogeneous solution $\boldsymbol{t} \in V_{k}^{N}$ exists, then the "shifted" vector $\boldsymbol{t}^{*} \in V_{k}^{N}$ as in (23) also satisfies the discrete equations. The assumption $N \bmod k=0$ in these theorems is not very restrictive: for a given $k$ we can always use mesh sizes such that $N$ is an integer multiple of $k$.

Remark 3 Related to the discrete problem the same comment as in Remark 2 holds. The results in the Theorems 3 and 4 do not depend on the specific form of the function $\alpha(\cdot)$. 


\subsection{Continuation strategy}

We solve the discrete nonlinear system (20) with a continuation method. The nonlinearity in the model stems entirely from the nonlinear boundary condition

$$
q_{F}\left(T_{F}\right)=C_{D}\left\{F_{1}-F_{2} H\left(C_{D} T_{F}-1\right)\right\} T_{F},
$$

with coefficients $C_{D}, F_{1}, F_{2}$ and Heaviside function $H(\cdot)$ according to Section 2. The model is linear if $q_{F}$ is a linear function of $T_{F}$. This motivates our introduction of the non-physical nonlinearity parameter $P$ below as additional continuation parameter. We define

$$
q_{F}\left(T_{F}, P\right):=C_{D}\left\{F_{1}-P F_{2} H\left(C_{D} T_{F}-1\right)\right\} T_{F}, \quad \text { for } 0 \leq P \leq 1 .
$$

For $P=0$ we have a linear boundary condition; for $P=1$ the original nonlinear condition is recovered. Figure 2 demonstrates the smooth transition of the boiling curve $q_{F}\left(T_{F}, P\right)$ from the linear state $(P=0)$ to the final nonlinear state $(P=1)$ in Figure $1 b$.

The discrete nonlinear problem $(20)$ with the $P$-dependent heat-transfer condition $(24)$ can be represented as

$$
\mathcal{G}(\boldsymbol{t}, P):=\left(\boldsymbol{K}+\boldsymbol{M}_{P}(\boldsymbol{t})\right) \boldsymbol{t}-\boldsymbol{g}=0 .
$$

Note that $\boldsymbol{M}_{P}(\boldsymbol{t})$ depends on $P$ via $\boldsymbol{M}_{P}(\boldsymbol{t})=\operatorname{diag}\left(\alpha_{P}\left(T_{j}\right)\right)_{0 \leq j \leq N}$ with $\alpha_{P}\left(T_{F}\right)=\frac{\Pi_{2}}{\Lambda} \frac{q_{F}\left(T_{F}, P\right)}{T_{F}}$ and $q_{F}\left(T_{F}, P\right)$ as in (24). For each $P \in[0,1]$ the set of homogeneous solutions (i.e. $\boldsymbol{t}=$ constant) of this system can be easily computed. Starting on a branch of homogeneous solutions we apply a continuation algorithm ${ }^{2}$ to $P \rightarrow \mathcal{G}(\boldsymbol{t}, P)=0$ and determine bifurcations points on the homogeneous branches from which branches of heterogeneous solutions originate. These strategies and the resulting bifurcation diagrams are discussed in the following.

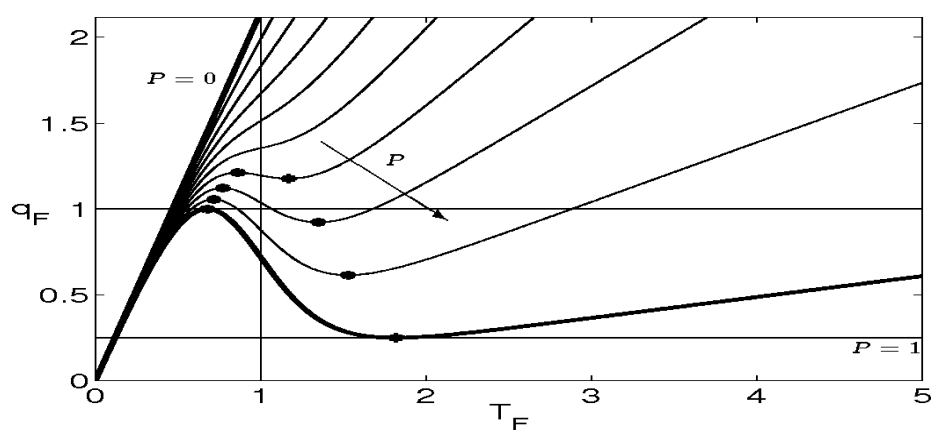

Figure 2: Controlling the degree of nonlinearity of the boiling curve via the nonlinearity parameter $P$. Shown is the smooth transition from a linear profile $(P=0)$ towards the physical boiling curve (heavy; $P=1$ ) with increasing $P$ (arrow). The stars denote the local maxima and minima that occur for $P$ beyond some non-zero lower limit.

\footnotetext{
${ }^{2}$ Here an in-house algorithm has been used, which is based upon techniques described in [22]. Elaboration on this continuation algorithm is beyond the present scope.
} 


\section{Numerical experiments: a representative case study}

In this section we consider the discrete steady-state problem for a fixed parameter set, namely for $\Lambda=0.2, D=0.2, \Pi_{1}=4, \Pi_{2}=2$ and $W=1$. The set of steady-state solutions is determined with the method explained in Section 4. Homogeneous solutions are obtained by means of a standard Newton-type root-finding algorithm applied to (8); heterogeneous solutions follow from continuation of the nonlinear system (25) in the nonlinearity parameter $P$. Note that for smooth boiling curves (i.e. $W>0$ ) the truncated Fourier expansion on which our discretization method is based exhibits exponential convergence and thus already for moderate values of $N$ the discrete problem (20) is a highly-accurate approximation of the continuous solution. In our experiments we use $N=128$.

\subsection{Homogeneous solutions}

The homogeneous steady-state solutions (9) are uniquely determined by the constant interface temperature $T_{F}$ as in (8). This interface temperature is given by the (multiple) intersection(s) of the boiling curve with the normalised heat supply $\bar{q}_{H} / Q_{C}=\Pi_{2}^{-1}$ (Figure $1 b$ ). For the determination of the physically-meaningful homogeneous solutions it is sufficient to solve (8) for $P=1$. However, to obtain bifurcation points from which branches of heterogeneous solutions originate we have to determine the homogeneous branches in the entire range $0 \leq P \leq 1$. These branches readily follow from solving (8) in this $P$-range. Two essentially different situations can be distinguished, as illustrated in Figure 3, namely: (i) one solution $T_{F}^{(1)}$ for $0 \leq P<P_{B}$ (Figure 3a); (ii) three solutions $\left(T_{F}^{(1)}, T_{F}^{(2)}, T_{F}^{(3)}\right.$ ) for $P_{B}<P \leq 1$ (Figure $3 c$ ). Both situations are connected through the degenerate case $P=P_{B}$, for which the local minimum of the boiling curve $q_{F}\left(\cdot, P_{B}\right)$ touches the normalised heat supply $\bar{q}_{H} / Q_{C}=\Pi_{2}^{-1}$, causing the second and third solutions to coincide (Figure $3 b$ ). Thus the system undergoes a qualitative change at $P=P_{B}$ through a so-called tangent bifurcation [21].
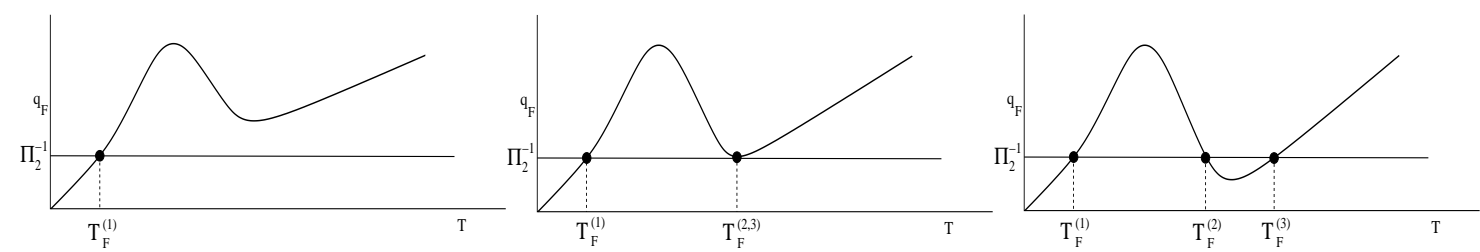

a) $0 \leq P<P_{B}$

b) $P=P_{B}$

c) $P_{B}<P \leq 1$

Figure 3: Homogeneous solutions to the nonlinear system as a function of the nonlinearity parameter $P$. Transition from the single-solution state (panel a) to the triple-solution state (panel c) occurs through a tangent bifurcation at $P=P_{B}$ (panel $b$ ).

The bifurcation diagram (Figure $4 a$ ) shows the solutions in terms of the functional $T_{\Sigma}=$ $\sum_{n} \widetilde{T}_{n}$ as a function of the nonlinearity parameter $P$. The heavy curves are the solution branches corresponding to the homogeneous solutions (9). The lower (nearly-horizontal) branch coincides with the intersection $T_{F}^{(1)}$ that exists for all $0 \leq P \leq 1$; the upper branch, with a turning point at $P_{B}$, coincides with the two intersections $T_{F}^{(2,3)}$ that exist only in the interval $P_{B} \leq P \leq 1$ (here $P_{B} \approx 0.926$ ). The lower and upper legs of this upper branch, connected at the turning point, correspond to $T_{F}^{(2)}$ and $T_{F}^{(3)}$, respectively. The solid curves 
are heterogeneous solution branches that originate from pitchfork bifurcations (dots) on the $T_{F}^{(2)}$-branch. An analysis of these bifurcation points is presented in the next section.

\subsection{Bifurcation points on branches of homogeneous solutions}

Bifurcations can only occur at $P$-values for which the Jacobian of $\mathcal{G}$ with respect to $\boldsymbol{t}$ is singular [22]. This Jacobian is given by

$$
\boldsymbol{J}=\frac{\partial \mathcal{G}}{\partial \boldsymbol{t}}=\boldsymbol{K}+\boldsymbol{Q}(\boldsymbol{t}), \quad \boldsymbol{Q}(\boldsymbol{t})=\operatorname{diag}\left(\gamma\left(T_{k}\right)\right)_{0 \leq k \leq N}, \quad \gamma(T):=\frac{\Pi_{2} \dot{q}_{F}(T)}{\Lambda} .
$$

On a homogeneous branch we have $\boldsymbol{t}=T_{F}(1, \ldots, 1)^{T}$ and thus $\boldsymbol{Q}(\boldsymbol{t})=\gamma\left(T_{F}\right) \boldsymbol{I}$, with $\boldsymbol{I}$ the identity matrix and $T_{F}$ the homogeneous interface temperature. Hence we obtain

$$
\boldsymbol{J}=\boldsymbol{K}+\gamma\left(T_{F}\right) \boldsymbol{I}=\boldsymbol{V} \boldsymbol{K}_{S} \boldsymbol{V}^{-1}+\gamma\left(T_{F}\right) \boldsymbol{I}=\boldsymbol{V} \boldsymbol{J}_{S} \boldsymbol{V}^{-1},
$$

with $\boldsymbol{J}_{S}=\operatorname{diag}\left(n \pi \tanh (n \pi D)+\gamma\left(T_{F}\right)\right)_{0 \leq n \leq N}$. Thus on the homogeneous branches we have an explicit eigenvector decomposition of the Jacobian. The eigenvalues $\lambda_{n}$ and corresponding eigenvectors $\boldsymbol{v}_{n}$ are given by

$$
\lambda_{n}=n \pi \tanh (n \pi D)+\gamma\left(T_{F}\right), \quad \boldsymbol{v}_{n}=\left(\cos \left(n \pi x_{0}\right), \ldots, \cos \left(n \pi x_{N}\right)\right)^{T}, \quad 0 \leq n \leq N .
$$

The eigenvector $\boldsymbol{v}_{n}$ coincides with the $n$-th Fourier mode. The Jacobian is singular if one or more of its eigenvalues $\lambda_{n}$ vanish. Because $n \pi \tanh (n \pi D) \geq 0$ for all $n \geq 0$, this can only happen if $\gamma\left(T_{F}\right) \leq 0$. Thus a bifurcation on a homogeneous solution branch can only occur for those $T_{F}$ for which the boiling curve has a negative slope $\left(\dot{q}_{F} \leq 0\right)$. From Figure 3 it follows that only intersection $T_{F}^{(2)}$ satisfies this criterion, thus explaining why bifurcations are restricted to the $T_{F}^{(2)}$-branch in the bifurcation diagram (Figure $4 a$ ). This implies that bifurcations - and thus multiple (heterogeneous) solutions - can only occur for interface temperatures in the transition range of the boiling curve.

Figure $4 b$ displays $\gamma$ (heavy curve) as a function of $P$ on the $T_{F}^{(2)}$-branch together with $F(n)=-n \pi \tanh (n \pi D)$ (dashed lines) for various $n$. The intersections $\gamma=F(n)$ correspond to $\lambda_{n}=0$. These eigenvalues are simple and the corresponding eigenspace is one-dimensional. From basic results in analysis (e.g., Thm. 28.3 in [23]) it follows that at these $P$-values the system indeed undergoes a bifurcation. These four $P$-values correspond to the positions of the bifurcations (filled circles) on the $T_{F}^{(2)}$-branch (Figure $4 a$ ). From Figure $4 b$. we see that for the case considered in this section the system has $\lambda_{n}=0$ only for $n=0,1,2,3$. The bifurcations on the $T_{F}^{(2)}$-branch in Figure $4 a$ correspond from left to right to the cases $n=0$, $n=1, n=2$ and $n=3$. This ordering results from the monotonic dependence of $\gamma$ on $P$. It also follows that in the range $P \in[0,1]$ bifurcation points on the homogeneous $T_{F}^{(2)}$-branch with constant temperatures $T_{F, P}$ occur for those wave numbers $n=0,1,2, \ldots$ that satisfy the inequality $n \pi \tanh (n \pi D)+\gamma\left(T_{F, 1}\right) \leq 0$.

\section{$5.3 \quad$ Heterogeneous solutions}

The eigenvectors $\boldsymbol{v}_{n}$ belonging to eigenvalues $\lambda_{n}$ correspond following (28) with individual Fourier modes. This implies that for $n \geq 1$ a heterogeneous discrete solution of the form

$$
T_{F}\left(\boldsymbol{x}_{\boldsymbol{j}}\right)=T_{F}^{(2)}+\epsilon \cos \left(n \pi x_{j}\right), \quad 0 \leq j \leq N, \epsilon \downarrow 0,
$$




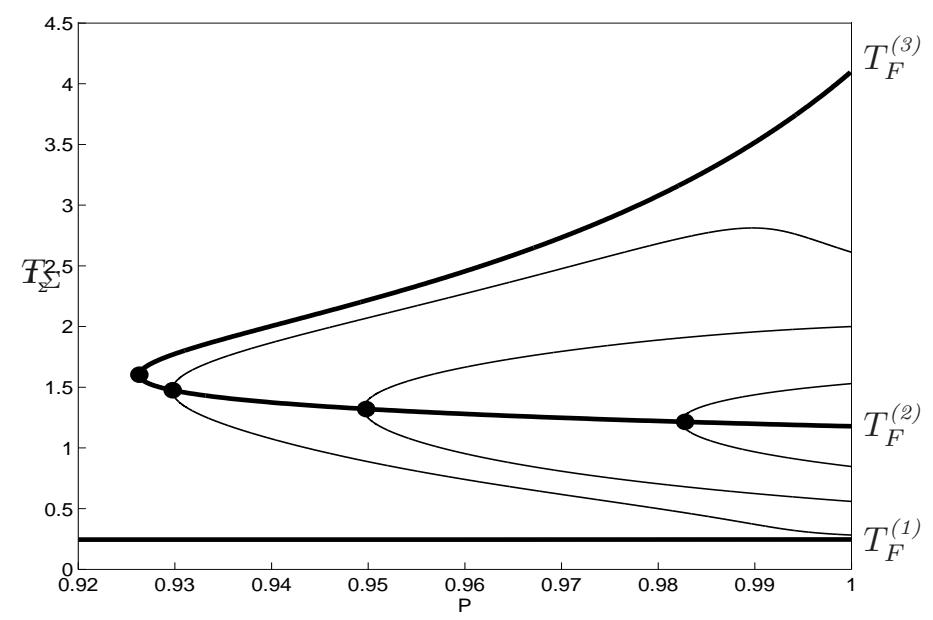

a) Bifurcation diagram for $P$.



b) Mechanism underlying bifurcations.

Figure 4: Bifurcations corresponding with the nonlinearity parameter P. Bifurcation diagram for $P$ at $\Lambda=0.2, D=0.2, \Pi_{1}=4, \Pi_{2}=2$ and $W=1$ (panel a). Heavy curves correspond to homogeneous solutions; solid curves correspond to heterogeneous solutions. Filled circles represent bifurcations. Mechanism underlying bifurcations (panel b): intersections of $\gamma$ along the homogeneous $T_{F}^{(2)}$-branch (heavy) with $F(n)$ (dashed).

emerges from the bifurcation point corresponding to $\lambda_{n}$ (Thm. 28.3 in [23]). The symmetry property derived in Section 4.1 implies pairs of heterogeneous solutions

$$
T_{F} \quad \text { as in }(29), \quad T_{F}^{*}(x)=T_{F}\left(x+\frac{1}{n}\right) .
$$

(Where $T_{F}^{*}$ is a discrete solution in the sense as explained in Theorem 4). The first bifurcation ( $n=0$; turning point on the $T_{F}^{(2)}$-branch) corresponds with a superimposed zero-th Fouriermode $\boldsymbol{v}_{0}$, hence the solution that emerges from this bifurcation point is homogeneous. Thus the first bifurcation point $(n=0)$ is of tangent type (shown schematically in Figure $3 b$ ) resulting in two homogeneous solutions. The bifurcations for $n>0$ involve non-coinciding superimposed modes that, due to symmetry properties (Theorems 2 and 4), form conjugate pairs of heterogeneous solutions; hence for $n>0$ we have pitchfork bifurcations.

The bifurcation diagram (Figure $4 a$ ) shows the solution branches as a function of the parameter $P$. Figure $5 a$ gives the evolution of the heterogeneous solutions near the first pitchfork bifurcation $(n=1 ; P=0.9297)$ for several $P$-values slightly larger than $P=0.9297$.

The final (i.e. physically-meaningful) heterogeneous states at $P=1$ are shown in Figure $5 b$. At this pitchfork bifurcation the heterogeneous solutions originate from the first non-constant Fourier mode (i.e. $n=1$ in (30)), which can be clearly seen in Figure $5 a$. Figure $5 b$ shows that the form of this first Fourier mode is roughly maintained throughout the evolution from the pitchfork bifurcation to the final state at $P=1$. For the second $(n=2)$ and third $(n=3)$ bifurcations similar behaviour occurs. The heterogeneous solutions emerge from the respective bifurcations at $P=0.9495$ and $P=0.9824$ in a manner akin to that shown in Figure $5 a$. The final states $(P=1)$ are given in Figures $5 c$ and $5 d$. The final states at $P=1$ are the physically-meaningful heterogeneous steady-state solutions at the heater-fluid interface $\Gamma_{F}$. These solutions are denoted mode-n solutions (with $n \in\{1,2,3\}$ ). Figure 6 


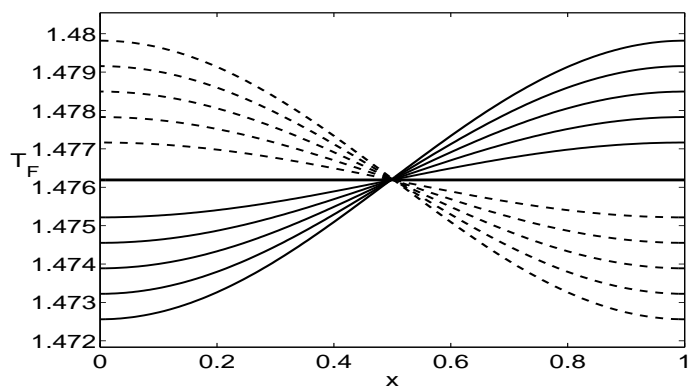

a) First pitchfork bifurcation $(P=0.9297)$.

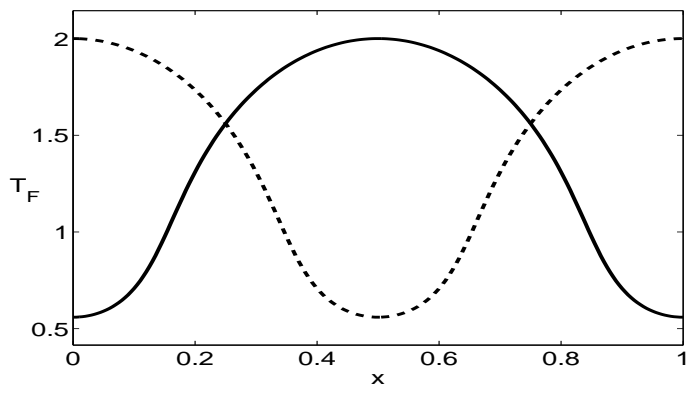

c) Final state second bifurcation $(P=0.9495)$.

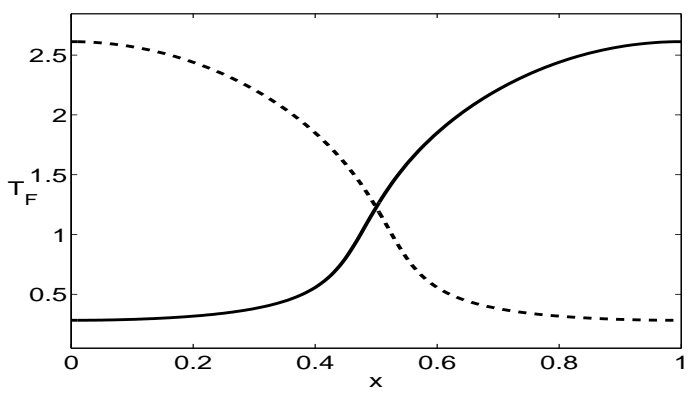

b) Corresponding final state $(P=1)$.

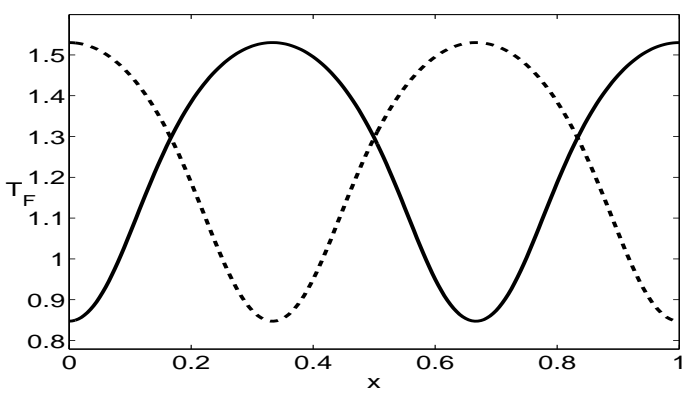

d) Final state third bifurcation $(P=0.0 .9824)$.

Figure 5: Origin of heterogeneous solutions. Panel a demonstrates the emergence from the first pitchfork bifurcation at $P=0.9297$ (heavy line: bifurcating homogeneous solution; solid and dashed curves: pair of heterogeneous solutions); panel $b$ gives the corresponding final state $(P=1)$. Panels $b$ and $c$ give the final states $(P=1)$ for second $(P=0.9495)$ and third $(P=0.0 .9824)$ pitchfork bifurcations, respectively. The final states are solutions of $(20)$.

shows the temperature distributions in the heater corresponding to these mode- $n$ solutions, revealing that the heterogeneous features occur mainly in lateral (i.e. $x$-wise) direction.

An important feature is the following. The mode- $n(n \in\{1,2,3\})$ solution $T_{F}$ emerges from a perturbation $\boldsymbol{v}_{n} \in V_{n}$. In a neighbourhood of the bifurcation point we have a heterogeneous solution $T_{F} \in V_{n}$ and thus, due to Theorem 4, also a dual solution $T_{F}^{*}$ with the symmetry property $T_{F}^{*}(x)=T_{F}\left(x+\frac{1}{n}\right)$. It turns out that during the continuation from the bifurcation point $P=P_{\text {bifur }}$ to the final value $P=1$ the heterogeneous solution $T_{F}$ remains in $V_{n}$ and thus the symmetry property $T_{F}^{*}(x)=T_{F}\left(x+\frac{1}{n}\right)$ holds for the whole range $P \in\left[P_{\text {bifur }}, 1\right]$.

Remark 4 An explanation for this "conservation of symmetry" property (for $P \in\left[P_{\text {bifur }}, 1\right]$ ) is the following. From Theorem 3 and Remark 3 it follows that the operator $\mathcal{G}(\boldsymbol{t}, P)$ has the property $\mathcal{G}(\cdot, P): V_{k}^{N} \rightarrow V_{k}^{N}$ for all $P \in[0,1]$ and all $k \geq 1$ such that $N \bmod k=0$. Note that a homogeneous solution lies in $V_{k}^{N}$ for all $k$. Let $P=P_{n}$ be such that $\lambda_{n}=0(n \geq 1)$ holds, i.e. $P_{n}$ corresponds to a mode- $n$ bifurcation point. We take $N$ such that $N \bmod n=0$ is satisfied. In a neighbourhood of $P_{n}$ we can consider

$$
\mathcal{G}(\cdot, P): V_{n}^{N} \rightarrow V_{n}^{N} .
$$

Starting from the bifurcation point we then have for increasing $P$ a heterogeneous solution, starting from $T_{F}^{(2)}+\epsilon \boldsymbol{v}_{n} \in V_{n}^{N}$ (with $\epsilon=P-P_{n}$ ), which due to (31) remains in $V_{n}^{N}$, until a new singularity is encountered. Thus during the continuation the solution $T_{F}=T_{F, P}$ remains in $V_{n}^{N}$ and has a dual solution with the symmetry property $T_{F}^{*}(x)=T_{F}\left(x+\frac{1}{n}\right)$ (Theorem 4 ). 


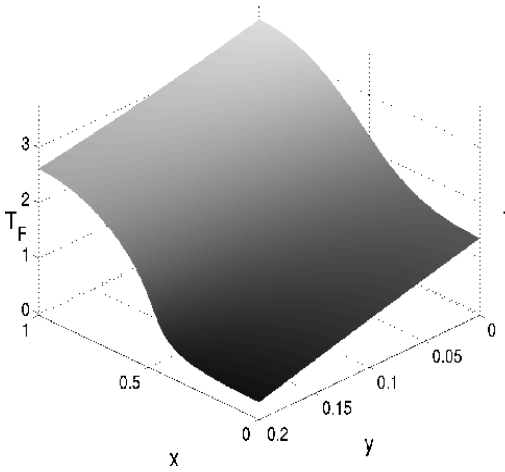

a) Mode-1 solution.

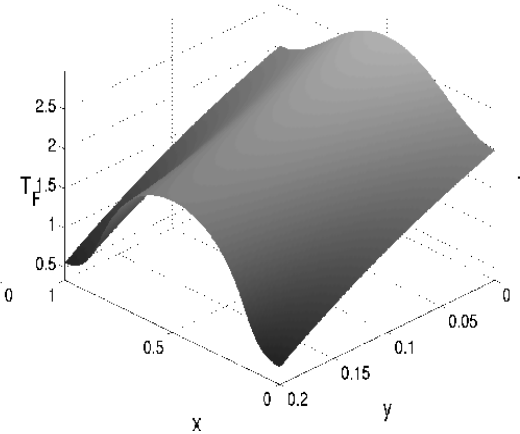

b) Mode-2 solution.

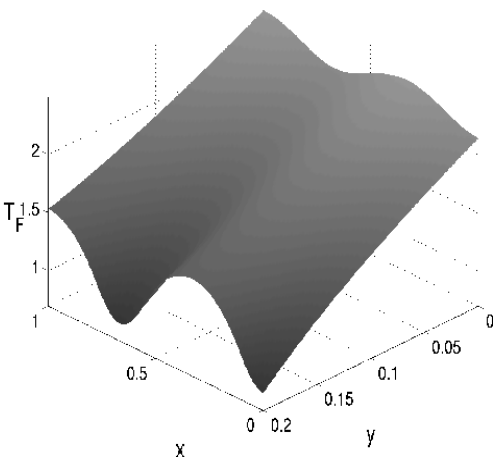

c) Mode-3 solution.

Figure 6: Temperature distributions $T(x, y)$ for the mode-n solutions. Heat supply and heat extraction is through the lines $y=0$ and $y=0.2$, respectively.

First results of a stability analysis show that all heterogeneous solutions as well as the homogeneous solution $T_{F}^{(2)}$ are unstable in time. Only the homogeneous solutions in the nucleate $\left(T_{F}^{(1)}\right)$ and film $\left(T_{F}^{(3)}\right)$ boiling regimes are stable. Slightly-perturbed heterogeneous mode- $n$ solutions converge to one of the two homogeneous solutions $T_{F}^{(1)}$ or $T_{F}^{(3)}$. The time scale for which the mode- $n$ heterogeneity remains clearly visible in the unsteady solution is significant for small $n$ and decreases rapidly for larger $n$.

\section{Numerical experiments: variation of system parameters}

The steady-state pool boiling model is determined by the system parameters $\left(\Lambda, D, \Pi_{1}, \Pi_{2}, W\right)$, cf. Section 2. These parameters control the following physical phenomena: (i) fluid-heater interaction via the boiling curve $\left(\Pi_{1}\right.$ and $W$ ); (ii) properties of the heater $(\Lambda$ and $D)$; (iii) heating conditions $\left(\Pi_{2}\right)$. Changes in the steady-state behaviour due to variation of these parameters is investigated below. We first perform a bifurcation analysis for the system parameters similar to that for the continuation parameter $P$ (Section 6.1). Then we study the physical changes in steady-state solutions with changing parameter values (Section 6.2). The bifurcation analysis is carried out with the continuation procedure proposed in Section 4, using the steady-state solutions determined in Section 5 as initial conditions.

\subsection{Bifurcation analysis for the system parameters}

\section{Effect of changes in the boiling curve: variation of $W$ and $\Pi_{1}$}

For an arbitrary transition width $W$ homogeneous steady-state solutions $T_{F}$ are given as solution of $q_{F}\left(T_{F}\right)=\Pi_{2}^{-1}$. From the shape of the boiling curve $q_{F}$ it is clear that variation of $W$ does not result in qualitative changes in the set of homogeneous solutions. We illustrate this in Figure $7 a$ for the boiling curves with $W=1$ (heavy), $W=0.1, W=0.5$ (solid) and corresponding homogeneous solutions $T_{F}^{(1,2)}$ (dots) for $\Pi_{2}=2$. (Note that the third intersection $T_{F}^{(3)}$ is not shown.) Heterogeneous mode- $n$ solutions originate from bifurcations on the $T_{F}^{(2)}$-branch occurring when $F(n)=\gamma\left(T_{F}^{(2)}\right)$ holds (Section 5.2). Figure $7 b$ shows $F(n)$ and $\gamma\left(T_{F}^{(2)}\right)$ for $0.5 \leq W \leq 1$ and $n \geq 1$. The boiling curve becomes steeper for smaller $W$-values, resulting in decreasing $\gamma$-values. This causes the number of intersections (and thus 
bifurcations) to increase monotonically with decreasing $W$. Narrowing the transition region thus progressively augments the number of mode- $n$ solutions and increases the wave-number range $1 \leq n \leq n_{\max }$ for which heterogeneous solutions exist. For $W=1$ three intersections occur and thus we have $n_{\max }=3$; for $W=0.5$ and $W=0.1$ the wave-number ranges increase to $n_{\max }=5$ and $n_{\max }=21$, respectively.

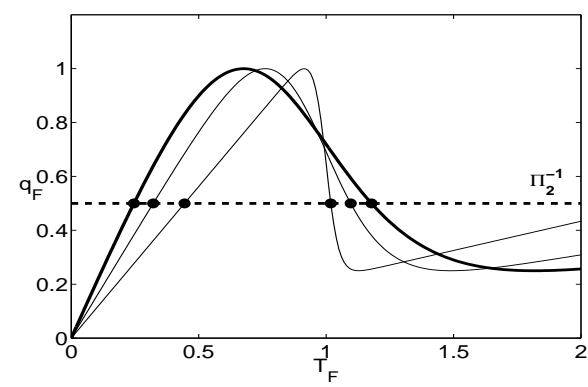

a) $q_{F}\left(T_{F}\right)$ for $W \in\{0,0.5,0.1\}$.

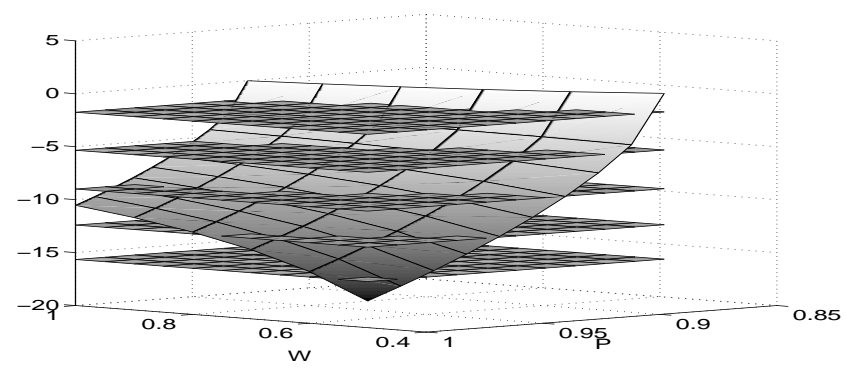

b) Intersections $F(n) \cap \gamma\left(T_{F}\right)$.

Figure 7: Effect of changing transition width $W$. Panel a illustrates the qualitative invariance of the solutions of $q_{F}(T)=\Pi_{2}^{-1}$ (dots) w.r.t. variation in $W$. The heavy curve is the boiling curve for the case $W=1$. The other curves are for $W=0.5$ and $W=0.1$. Panel $b$ shows $F(n)$ for $n>0$ (parallel planes) and $\gamma$ (surface) as a function of $P$ and $W$. The number of intersections $F(n) \cap \gamma$ (implying mode-n solutions) grows with decreasing $W$.

Figure $8 a$ shows the bifurcation diagram for parameter $P$ in case of $W=0.5$. (Compare with the case $W=1$ in Figure $4 a$.) Figure $8 b$ shows the $T_{F}^{(2)}$-branches and corresponding pitchfork bifurcation points (dots) for the transition widths $W=(0.1,0.2,0.5,1)$.

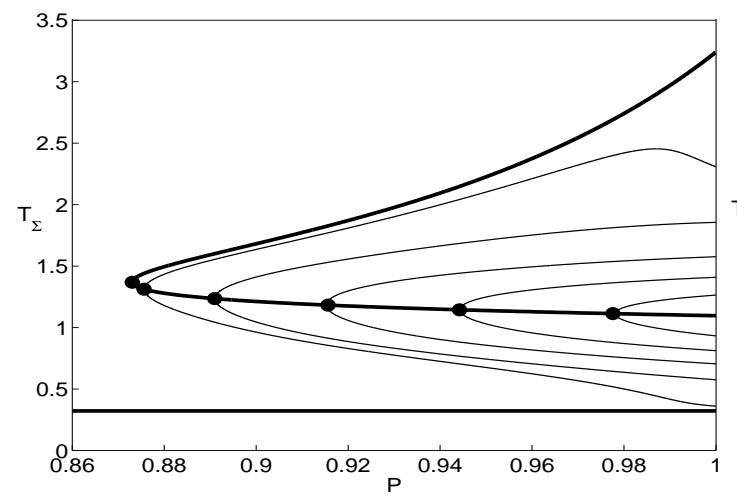

a) Bifurcation diagram for $W=0.5$.

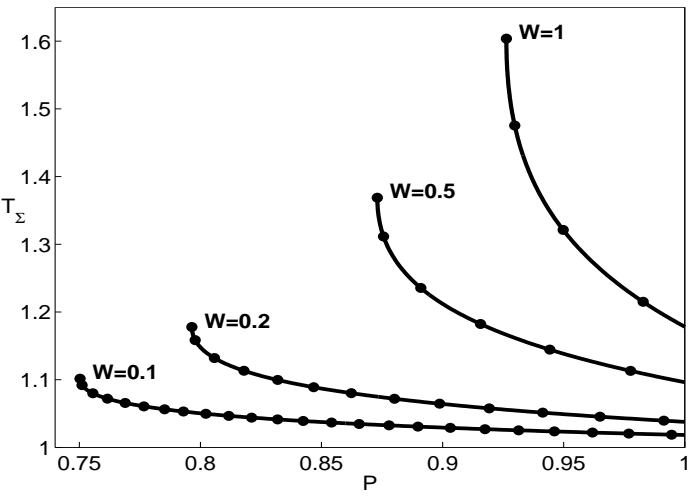

b) $T_{F}^{(2)}$-branch for several $W$ values.

Figure 8: Set of mode-n solutions for decreasing transition width $W$. Panel a shows the bifurcation diagram for $W=0.5$. Panel $b$ shows the $T_{F}^{(2)}$-branches and corresponding pitchfork bifurcations (dots) for the indicated transition widths $W$. 
The heat-flux ratio $\Pi_{1}$ influences the boiling curve in a manner comparable to the continuation parameter $P$ and changes thus have similar effects. This is demonstrated in Figure $9 a$ for the case of a physical boiling curve $(P=1)$ with transition width $W=1$. Homogeneous solutions correspond to intersections $q_{F}\left(T_{F}\right)=\Pi_{2}^{-1}$, which occur according to the scenario sketched for increasing $P$ in Figure 3. Increasing $\Pi_{1}$ lowers the local minimum and consequently causes a transition from one $\left(\Pi_{1}<\Pi_{2}\right)$ to three $\left(\Pi_{1}>\Pi_{2}\right)$ solutions via the tangent bifurcation at $\Pi_{1}=\Pi_{2}$. On the $T_{F}^{(2)}$-branch a pitchfork bifurcation occurs if $F(n)=\gamma\left(T_{F}^{(2)}\right)$ holds. In Figure $9 b$ the graphs of $F(n)$ and $\gamma\left(T_{F}^{(2)}\right)$ as a function of $\Pi_{1}$ and $W$ are given. Note the similarity between Figure $9 b$ and Figure $7 b$. It follows that $\gamma\left(T_{F}^{(2)}\right)$ decreases monotonically with increasing $\Pi_{1}$. Hence, the number of intersections $F(n)=\gamma\left(T_{F}^{(2)}\right)$ (and thus of mode- $n$ solutions) increases with increasing $\Pi_{1}$. The bifurcation diagram for $\Pi_{1}$ is qualitatively similar to that for $P$ (Figure $4 a$ ). Furthermore, increasing $\Pi_{1}$ has a effect similar to decreasing the transition width $W$ : The wave-number range in which bifurcations exist is enlarged.

The above reveals that variations of $W$ and $\Pi_{1}$ (in realistic ranges) do not lead to qualitative changes in the behaviour of the system. Therefore it is no severe restriction to consider only one fixed boiling curve. In the remainder we use as default the boiling-curve parameters $\Pi_{1}=4$ and $W=1$ and investigate the role of the heating conditions $\left(\Pi_{2}\right)$ and heater properties $(\Lambda$ and $D)$ on the behaviour of the pool boiling model.

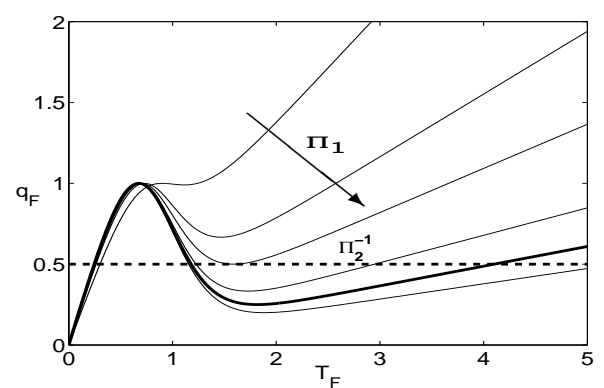

a) Boiling curve for several $\Pi_{1}$ values.

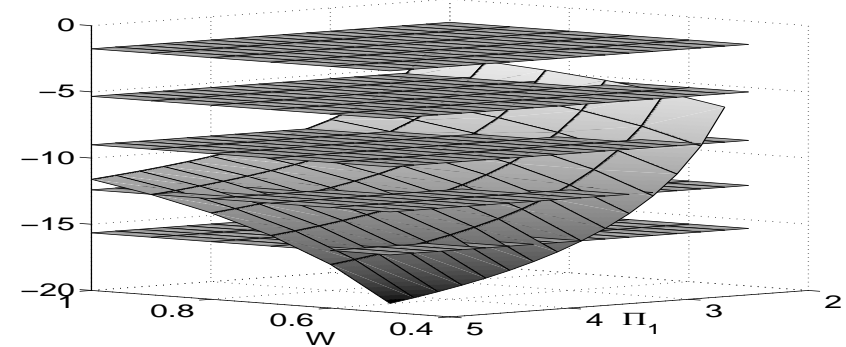

b) Intersections $F(n) \cap \gamma\left(T_{F}\right)$.

Figure 9: Effect of changing heat ratio $\Pi_{1}$ on the steady-state behaviour. Panel a shows the boiling curve as a function of $\Pi_{1}$ (cf. Figure 2). The heavy curve is the boiling curve for $W=1$. Panel $b$ shows $F(n)$ for $n>0$ (parallel planes) and $\gamma$ as a function of $W$ and $\Pi_{1}$. The number of intersections $F(n) \cap \gamma$ (implying mode- $n$ solutions) grows with increasing $\Pi_{1}$.

\section{Effect of changes in the heating conditions: variation of $\Pi_{2}$}

The heating conditions $\left(\Pi_{2}\right)$ determine the regions of existence of multiple solutions and thus the global boiling mode. Multiple (heterogeneous) solutions are restricted to the regime $1 \leq \Pi_{2} \leq \Pi_{1}$. Outside the range $1 \leq \Pi_{2} \leq \Pi_{1}$ only one homogeneous solution - and consequently only one homogeneous boiling state - exists, namely $T_{F}^{(1)}$ (nucleate boiling) for $\Pi_{2}>\Pi_{1}$ and $T_{F}^{(3)}$ (film boiling) for $0 \leq \Pi_{2}<1$. The link between multiplicity of steadystate solutions and heating conditions is consistent with laboratory experiments [6] and is a fundamental property of pool boiling systems.

Figure $10 a$ shows a typical bifurcation diagram for $\Pi_{2}$ with heater properties $\Lambda=0.2$ and $D=0.2$. The homogeneous branches are combined into the heavy curve and connected via 
the turning points at $\Pi_{2}=1$ and $\Pi_{2}=\Pi_{1}(=4)$. These turning points bound the parameter range $1 \leq \Pi_{2} \leq \Pi_{1}$ and are the tangent bifurcations that lead to multiple homogeneous solutions. The lower and upper sections of the combined homogeneous branch coincide with the $T_{F}^{(1)}$-and $T_{F}^{(3)}$-branches, respectively. The centre section corresponds to the $T_{F}^{(2)}$-branch. The heterogeneous branches of corresponding to the mode- $n$ solutions (solid) originate pairwise from this branch and form closed concentric loops, with $n$ increasing from $n=1$ in inward direction (here $n=1,2,3$ ). The dashed line indicates the case study of Section 5 .

Bifurcations on the homogeneous $T_{F}^{(2)}$-branch coincide with $\lambda_{n}=0$, with $\lambda_{n}$ as in (28), i.e. for $T_{F}=T_{F}\left(\Pi_{2}\right)$ such that $g\left(\Pi_{2}\right):=\Pi_{2} \dot{q}_{F}\left(T_{F}\left(\Pi_{2}\right)\right)=-\Lambda n \pi \tanh (n \pi D)$ holds. Parameters $\Lambda$ and $D$ are fixed: $\Lambda=D=0.2$. Hence, a bifurcation occurs if $\Pi_{2} \in[0,4]$ and $n \geq 1$ are such that $g\left(\Pi_{2}\right)=-\Lambda n \pi \tanh (n \pi D)$. From the profile of $g$ (not shown) it follows that solutions of the latter equation occur as pairs $\Pi_{2}^{L}(n), \Pi_{2}^{R}(n)$ with $1<\Pi_{2}^{L}(n)<\Pi_{2}^{R}(n)<4$. Thus mode- $n$ bifurcation points occur in pairs, which leads to the closed heterogeneous branches in Figure 10a. Furthermore, the function $n \rightarrow \Pi_{2}^{L}(n)\left(n \rightarrow \Pi_{2}^{R}(n)\right)$ is strictly increasing (decreasing). This explains the specific ordering of the heterogeneous branches.

The bifurcation diagram (Figure 10a) provides information on the qualitative steady-state behaviour of the pool boiling system as a function of the heating conditions. Regimes $\Pi_{2}>\Pi_{1}$ (nucleate boiling) and $0 \leq \Pi_{2}<1$ (film boiling) allow only one single solution branch. In the range $1 \leq \Pi_{2} \leq \Pi_{1}$ (transition boiling) multiple solution branches occur. Multiplicity phenomena increase (more mode- $n$ solutions) when $\Pi_{2}$ moves the borders of the interval $\left[1, \Pi_{1}\right]$ towards its centre. The multiple solution branches allow different steady states that the pool boiling system may have during transition from nucleate to film boiling (and vice versa) with changing heat supply.

\section{Effect of changes in the heater properties: variation of $\Lambda$ and $D$}

We take a fixed value $\Pi_{2}=2$ and vary $\Lambda$ and $D$. First we consider the effects of changing the non-dimensional thermal conductivity $\Lambda$, with $D=0.2$ fixed. The resulting bifurcation diagram is shown in Figure 10b. The heavy lines are the homogeneous branches (which do not depend on $\Lambda$ ); the solid curves are the heterogeneous branches originating from the $T_{F}^{(2)}$ branch. The dashed line refers to the case study of Section 5 . The wave number $n$ of the mode- $n$ solutions corresponding to the heterogeneous branches increases from $n=1$ (rightmost heterogeneous branch) monotonically with decreasing $\Lambda$. This ordering can be explained as follows. A bifurcation point on the $T_{F}^{(2)}$-branch occurs if the equality $\Lambda=-\frac{\Pi_{2} \dot{q}_{F}\left(T_{F}\right)}{n \pi \tanh (n \pi D)}$ holds, with $T_{F}$ such that $q_{F}\left(T_{F}\right)=\Pi_{2}^{-1}$ and $\dot{q}_{F}\left(T_{F}\right) \leq 0$. This homogeneous solution $T_{F}$ does not depend on $\Lambda$. Therefore, since $D$ is also constant, for every $n \geq 1$ there is a unique solution $\Lambda(n)$ to this equality. Furthermore, the function $n \rightarrow \Lambda(n)$ decreases strict monotonically.

We now consider the effect of changes in the aspect ratio $D$, with a fixed $\Lambda=0.2$. The corresponding bifurcation diagram is shown in Figure 10c. Heavy and solid lines again indicate homogeneous and heterogeneous branches; the dashed line refers to the case study of Section 5. This diagram resembles that for $\Lambda$ in Figure $10 b$ in that homogeneous branches do not depends on $D$ and heterogeneous branches correspond from right to left with mode- $n$ solutions with increasing wave number $n$. However, bifurcation points occur only for wave numbers $n \geq 4$. For given $\Lambda$ and $\Pi_{2}$ the solutions corresponding to the wave numbers $1 \leq n \leq 3$ exist for any $D>0$ and thus do not undergo bifurcations in this parameter range. This can be explained by similar arguments as used above for $\Lambda$. At a bifurcation point on the $T_{F}^{(2)}$ branch the equality $\tanh (n \pi D)=-\frac{\Pi_{2} \dot{q}_{F}\left(T_{F}\right)}{n \pi \Lambda}$ is fulfilled, with $T_{F}$ such that $q_{F}\left(T_{F}\right)=\Pi_{2}^{-1}$ 
and $\dot{q}_{F}\left(T_{F}\right) \leq 0$. This homogeneous solution $T_{F}$ does not depend on $D$. For $n$ too small $(1 \leq n \leq 3)$ we have $-\frac{\Pi_{2} \dot{q}_{F}\left(T_{F}\right)}{n \pi \Lambda} \geq 1$ and thus the equality does not have a solution. For $n$ sufficiently large $(n \geq 4)$ we have $0<-\frac{\Pi_{2} \dot{q}_{F}\left(T_{F}\right)}{n \pi \Lambda}<1$ and thus the equality has a unique solution $D(n)$. One easily verifies that $n \rightarrow D(n)$ is strictly decreasing. For $\Lambda$ sufficiently

large we have $0<-\frac{\Pi_{2} \dot{q}_{F}\left(T_{F}\right)}{n \pi \Lambda}<1$ for all $n \geq 1$ and then we have mode- $n$ bifurcation points on the homogeneous $T_{F}^{(2)}$-branch for all $n \geq 1$.

The above reveals that both with decreasing $\Lambda$ and $D$ the number of bifurcation points occurring increases. Since each new bifurcation implies a new pair of mode- $n$ solutions, this implies that decreasing thermal conductivity $(\Lambda)$ and/or relative heater thickness $(D)$ induces more multiplicity and heterogeneity phenomena in our pool boiling model. Conversely, increasing thermal conductivity and/or relative heater thickness enhances homogeneity.

This effect of heater thickness is known from laboratory experiments [24]. However, an essential difference between the effects of variation in $\Lambda$ and in $D$ is that increasing thermal conductivity at some point always causes vanishing of heterogeneous solutions, whereas for specific ranges of $\Lambda$ (and $\Pi_{2}$ ) heterogeneous solutions are always present, irrespective of heater thickness. The bifurcation diagram in Figure $10 c$ indicates that in this particular range of $\Lambda$ and $\Pi_{2}$ bifurcations occur only for mode- $n$ solutions with $n \geq 4$; solutions for $n=(1,2,3)$ exist for any aspect ratio $D$ here.

\subsection{Physical changes in steady-state solutions}

Mode- $n$ bifurcations occur in $\Lambda$ - and $D$-directions if for a certain wave number $n$ the eigenvalue $\lambda_{n}$ of the Jacobian equals zero. Moving away from the bifurcation, by decreasing the system parameter, amplifies the heterogeneous features of the corresponding mode- $n$ solutions in a similar way as demonstrated in Figure 5 for the continuation parameter $P$.

Figure $11 a$ shows the change of the interface temperature $T_{F}(x)$ of the mode- 1 solution with decreasing thermal conductivity $\Lambda$ (solid curves) from its nearly homogeneous state at the bifurcation (heavy line; $\Lambda=1.20$ ) to its state just above the lower bound $\Lambda=0$ (heavy curve; $\Lambda=0.05$ ). The plot reveals a progressive steepening of the profile with decreasing $\Lambda$ to such an extent that it suggests a discontinuous profile for $\Lambda \downarrow 0$. This steepening results from the higher temperature gradients caused by the higher resistance to heat conduction due to lower thermal conductivity. For vanishing heat conduction this implies locally-infinite temperature gradients and consequently discontinuous profiles.

Figure $11 b$ shows the change of $T_{F}(x)$ of the mode- 4 solution (the lowest mode- $n$ solution that originates from a bifurcation here) with decreasing aspect ratio $D$. The plot reveals that decreasing the relative heater thickness amplifies, similar to decreasing the thermal conductivity, the heterogeneous features of the mode- $n$. The progression suggests that, in contrast to the behaviour found for $\Lambda$, the solution now tends to a smooth profile for vanishing aspect ratio $D$. The heavy curve in Figure $11 b$ corresponds to $D=0.01$ and is believed to be a good approximation for the profile associated with the 'thin' heater $(D=0)$.

In $\Pi_{2}$-direction, mode- $n$ bifurcations occur pair-wise and thus result in essentially nonmonotonic dependence of the solution on changing heating conditions (not shown). Here the homogeneous solution corresponding with $\Pi_{2}=\Pi_{2}^{L}$ (left bifurcation point) develops heterogeneous features that are amplified with increasing $\Pi_{2}$ in a way akin to that shown in Figure 11, up to some value $\Pi_{2}=\Pi_{2 r e v}$. The latter marks the point at which the process reverses and the heterogeneous features progressively diminish with further increasing $\Pi_{2}$ until 


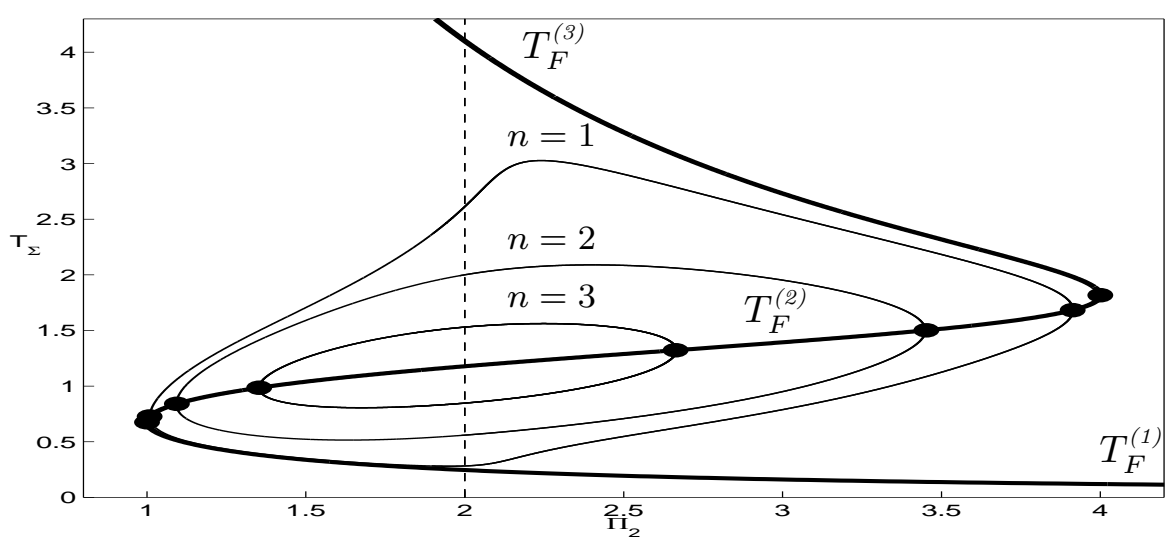

a) Bifurcation diagram for $\Pi_{2}$.

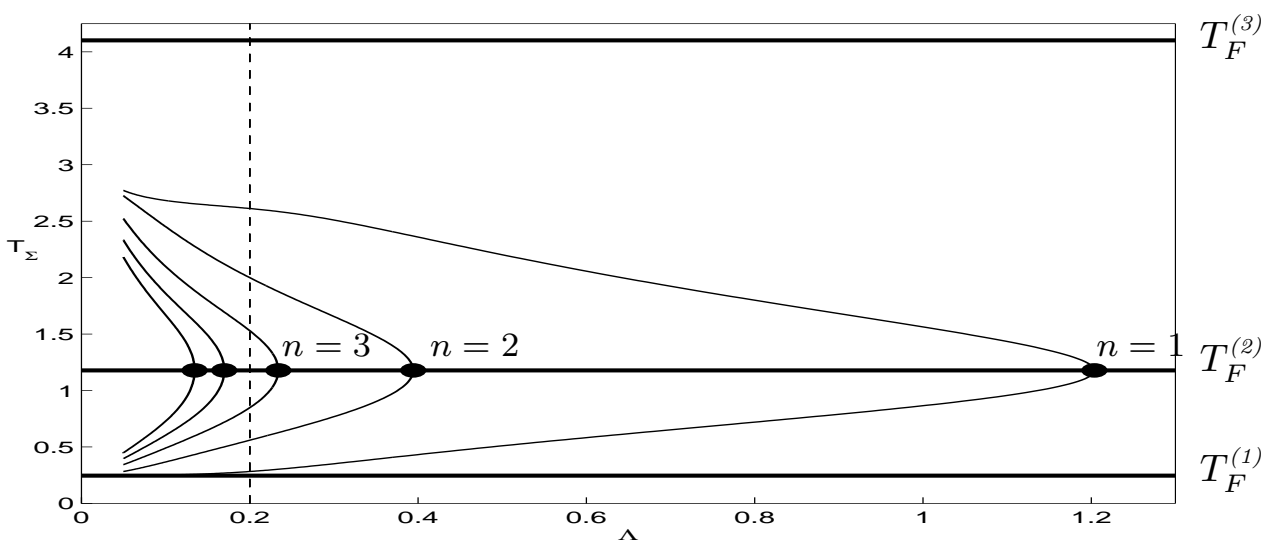

b) Bifurcation diagram for $\Lambda$.

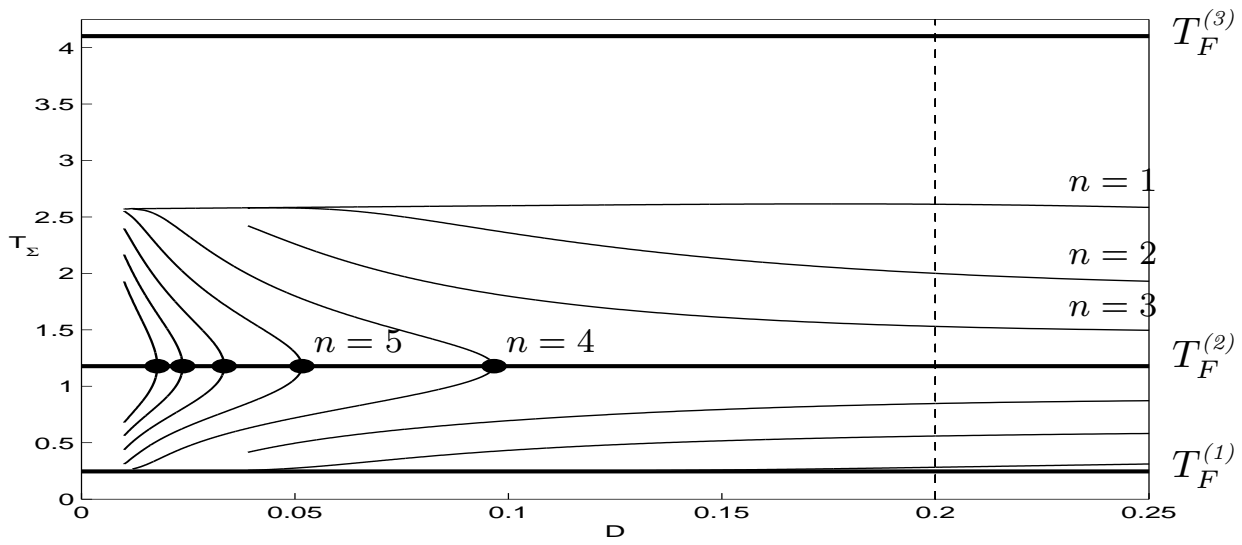

c) Bifurcation diagram for $D$.

Figure 10: Bifurcation diagrams for system parameters $\Pi_{2}(\Lambda=0.2, D=0.2), \Lambda\left(\Pi_{2}=2, D=\right.$ $0.2)$ and $D\left(\Lambda=0.2, \Pi_{2}=2\right)$. The intersections of the solution branches with the vertical dashed lines in the bifurcation diagrams for $\Lambda$ and $\Pi_{2}$ coincide with the solutions at $P=1$ in Figure $4 a$. 
the homogeneous solution corresponding with $\Pi_{2}=\Pi_{2}^{R}$ (right bifurcation point) is reached.

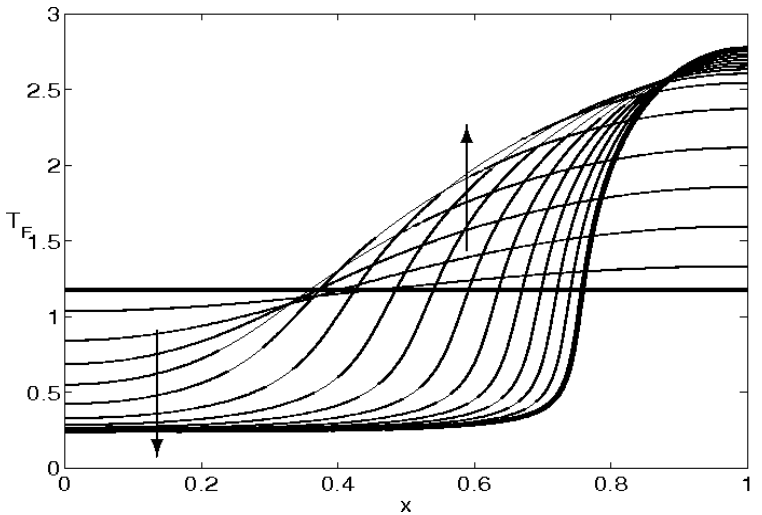

a) Mode-1 solution for $\Lambda$

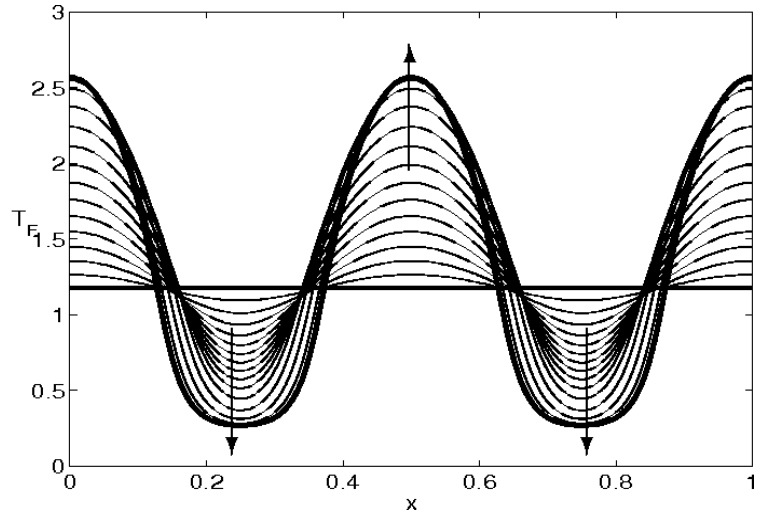

b) Mode-4 solution for $D$

Figure 11: Changes in interface profile of indicated mode-n solutions as a function of $\Lambda$ (panel a) and D (panel b). The heavy horizontal lines are the profiles at the bifurcation; the heavy curves are the profiles farthest away from the bifurcation. The solid curves are the intermediate states. The arrows indicate progression with decreasing parameter $\Lambda$ or $D$.

\section{Conclusions}

In this paper we consider a two-dimensional heat equation with a nonlinear Neumann boundary condition on part of the boundary as a simple model for $2 \mathrm{D}$ pool boiling processes. The nonlinear Neumann boundary condition models the heat flux from the heater to the fluid by means of a nonlinear local heat-flux temperature relation that is similar to the boiling curve. A key issue is the existence of multiple steady-state solutions with heterogeneous interface temperature. The seperation of variables technique leads to a reduction of the twodimensional problem to a one-dimensional problem for the temperature at the heater-to-fluid interface, cf. (7). The latter problem is discretised using a collocation method. Both the continuous and discrete problem (at the interface) have a symmetry property (Theorems 2 and 4) that immediately implies multiplicity of heterogeneous solutions. These originate from bifurcations on a branch of homogeneous solutions. The existence of symmetries (Theorem 2) and the conservation of symmetries (Theorem 1) are two fundamental properties of the model. The multiple solution structure and its dependence on certain system parameters is studied through a bifurcation analysis applied to the discretised problem.

We outline the main conclusions from the analysis.

Multiple (heterogeneous) steady-state solutions exist. Multiplicity and heterogeneity are restricted to the transition-boiling regime; the nucleate-boiling and film-boiling regimes admit but one unique solution exists, which is always homogeneous. Heterogeneous solutions represent temperature distributions that correspond to nucleate and film boiling regions and thus are essentially two-mode boiling states. Heating conditions are modelled as a constant heat flux on the boundary opposite to the heater-fluid interface. Heat supply between critical heat flux (CHF) and Leidenfrost heat flux (LHF; local minimum on boiling curve) implies three homogeneous solutions, each in one of the three boiling regimes. Heat supply outside this 
range results in only homogeneous solution, corresponding to either nucleate or film boiling. Heterogeneous solutions originate pairwise from pitchfork bifurcation points on the branch of homogeneous solutions in the transition regime of the boiling curve and can occur only under specific heating conditions. The actual steady-state solution attained at such pitchfork bifurcations is inherently unpredictable. Relevant system parameters are boiling curve coefficients (transition width $W$; CHF-LHF ratio $\Pi_{1}$ ) and heater properties (aspect ratio $D$; thermal conductivity $\Lambda$ ). Decreasing (one of) $W, D$ and $\Lambda$ and/or increasing $\Pi_{1}$ enlarges the set of heterogeneous solutions, suggesting this induces stronger multiplicity and heterogeneity of solutions of the pool boiling problem. However, variation of these parameters does not cause fundamental changes in the steady-state behaviour of the system. Multiplicity and heterogeneity only show a quantitative dependence on these parameters. The essential condition is $1 \leq \Pi_{2} \leq \Pi_{1}$. If this condition is satisfied, there exists a homogeneous solution in the transition range of the boiling curve, leading to bifurcating pairs of heterogeneous solutions.

Important phenomena resulting from numerical simulations of our model are consistent with properties known from laboratory experiments. This suggests that the model provides an (at least qualitatively) adequate description of pool boiling.

Both the present model and the numerical techniques used can be extended relatively straightforward to 3D pool boiling systems. This is a topic of current research [25]. Furthermore, results of a stability analysis of the steady-state solutions will be presented in a forthcoming paper. Preliminary results of this analysis reveal that steady-state solutions are always unstable, except for the two homogeneous solutions corresponding to the nucleate and film boiling regimes. Miscellaneous issues to be considered in future work may include the effect of different heating methods [26] and stabilisation via active control [7].

\section{Acknowledgment}

The presented work has benefitted greatly from the continuation algorithm kindly provided by Robert Grosch, Process Systems Engineering, RWTH Aachen.

\section{References}

[1] J. R. Thome, Boiling, in: A. Bejan, A. D. Krause (Eds), Handbook of Heat Transfer, Wiley \& Sons, Hoboken, 2003, pp. 635-717.

[2] I. Mudawar, Assessment of high-heat flux thermal management schemes, IEEE Transactions-CPMT: Components and Packaging Technologies, 24 (2001), 122-141.

[3] V. K. Dhir, Boiling Heat Transfer, Ann. Rev. Fluid Mech., 30 (1998), 365-401.

[4] T. G. Theofanous, J. P. Tu, A. T. Dinh, T. N. Dinh, The boiling crisis phenomenon. Part I: nucleation and nucleate boiling heat transfer, Exp. Therm. Fluid Sci., 26 (2002), $775-792$.

[5] V. K. Dhir, Nucleate and transition boiling heat transfer, Int. J. Heat Fluid Flow, 12 (1991), 290-314.

[6] H. Auracher, W. Marquardt, Heat transfer characteristics and mechanisms along entire boiling curves under steady-state and transient conditions, J. Heat Fluid Flow, 25 (2004), 223-242.

[7] H. Auracher, W. Marquardt, Experimental studies of boiling mechanisms in all boiling regimes under steady-state and transient conditions, Int. J. Therm. Sci., 41 (2002), $586-598$. 
[8] H. van Ouwekerk, Burnout in pool boiling. The stability of boiling mechanisms, Int. J. Heat Mass Transfer, 15 (1972), 25-33.

[9] S. A. Zhukov, V. V. Barelko, A. G. Merzhanov, Wave processes on heat generating surfaces in pool boiling, Int. J. Heat Mass Transfer, 24 (1980), 47-55.

[10] J. Blum, T. Lüttich, W. Marquardt, Temperature wave propagation as a route from nucleate to film boiling? in: G.P. Celata, P. DiMarco, R.K. Shah (Eds), Proceedings of the Second International Symposium on Two-Phase Flow Modelling and Experimentation, Rome, Vol 1, Edizioni ETS, Pisa, 1999.

[11] S. A. Zhukov, V. V. Barelko, Nonuniform steady states of the boiling process in the transition region between the nucleate and film regimes, Int. J. Heat Mass Transfer, 26 (1983), 1121-1130.

[12] B. A. Gabaraev, S. A. Kovalev, Yu. S. Molochnikov, S. L. Solov'ev, S. V. Usitakov, Rewetting and autowave change of boiling modes, High Temp., 39 (2001), 302-314.

[13] S. A. Kovalev, An investigation of minimum heat fluxes in pool boiling of water, Int. J. Heat Mass Transfer, 9 (1966), 1219-1226.

[14] S. A. Kovalev, G. B. Rybchinskaya, Prediction of the stability of pool boiling heat transfer to finite disturbances, Int. J. Heat Mass Transfer, 21 (1978), 691-700.

[15] S. A. Kovalev, S. V. Usitakov, Analysis of the stability of boiling modes involving the use of stability diagrams, High Temp., 41 (2003), 68-78.

[16] A. V. Gurevich, R. G. Mints, Self-heating in normal metals and superconductors, Rev. Mod. Phys. 59 (1987), 941-999.

[17] J. Blum, W. Marquardt, Objection to Haramura's criterions for temperature uniformity across the surface in transition boiling, Internal report LPT-1998-14 (1998), Lehrstuhl für Prozesstechnik, RWTH Aachen.

[18] M. F. M. Speetjens, Steady-state behaviour of 2D pool boiling problems, in: A. J. Nowak, R. A. Bialecki, G. Wecel (Eds), Proceedings Eurotherm seminar 82 Numerical Heat Transfer, 2005, pp. 923-931.

[19] E. Kreyszig, Advanced Engineering Mathematics, Wiley, Chichester, 1999.

[20] C. Canuto, M. Y. Hussaini, A. Quarteroni, T. A. Zang, Spectral Methods in Fluid Dynamics, Springer, Berlin, 1987.

[21] E. Ott, Chaos in Dynamical Systems, second ed., Cambridge University Press, Cambridge, 2002.

[22] W. J. F Govaerts, Numerical Methods for Bifurcations of Dynamical Equilibria, SIAM, Philadelphia, 2000.

[23] K. Deimling, Nonlinear Functional Analysis, Springer, Berlin, 1985.

[24] J. Blum, W. Marquardt, H. Auracher, Stability of Boiling Systems, Int. J. Heat Mass Transfer, 39 (1996), 3021-3033.

[25] M. Speetjens, A. Reusken, W. Marquardt, Steady-state solutions in a three-dimensional nonlinear pool-boiling heat-transfer model, IGPM report 263, RWTH-Aachen (2006).

[26] J. Darabi, M. M. Ohadi, M. A. Fanni, S. V. Dessiatoun, M. A. Kedzierski, Effect of heating boundary conditions on pool boiling experiments, HVAC\&R Research, 5 (1999), $1-14$. 


\section{A Proofs of Theorems 3 and 4}

We need the following lemma:

Lemma 1 Let $1 \leq k \leq N$ be such that $N \bmod k=0$. For $\boldsymbol{t} \in V_{k}^{N}$ we then have $M(\boldsymbol{t}) \boldsymbol{t} \in V_{k}^{N}$.

Proof. Define $m:=\frac{N}{k}$. For a vector $\boldsymbol{z} \in \mathbb{R}^{N+1}$ we use the notation $\boldsymbol{z}=\left(z_{0}, z_{1}, \ldots, z_{N}\right)^{T}$. For $\boldsymbol{t} \in V_{k}^{N}$ we have $\boldsymbol{t}=\sum_{n=0}^{m} \alpha_{n} \mathbf{v}_{k n}$ and thus $t_{i}=\sum_{n=0}^{m} \alpha_{n} \cos \left(k n \pi x_{i}\right)$ for $0 \leq i \leq N$. From this it follows that

$$
t_{m+i}=t_{m-i} \quad(0 \leq i \leq m), \quad t_{i+2 m}=t_{i} \quad(0 \leq i \leq N-2 m) .
$$

The vector $\boldsymbol{r}:=M(\boldsymbol{t}) \boldsymbol{t}$ has entries $r_{i}=\alpha\left(t_{i}\right) t_{i}$ and thus we have

$$
r_{m+i}=r_{m-i} \quad(0 \leq i \leq m), \quad r_{i+2 m}=r_{i} \quad(0 \leq i \leq N-2 m) .
$$

The vector $\left(r_{j}\right)_{0 \leq j \leq m}$ has a discrete Fourier transform, cf. (16), $r_{j}=\sum_{\ell=0}^{m} \tilde{r}_{\ell} \cos \left(\ell \pi \hat{x}_{j}\right)=$ $\sum_{\ell=0}^{m} \tilde{r}_{\ell} \cos \left(k \ell \pi x_{j}\right)$, with $0 \leq j \leq m$ and $\hat{x}_{j}:=\frac{j}{m}$. Due to $(32)$ and $\cos \left(k \ell \pi x_{m+j}\right)=$ $\cos \left(k \ell \pi x_{m-j}\right) \quad(0 \leq j \leq m), \cos \left(k \ell \pi x_{j+2 m}\right)=\cos \left(k \ell \pi x_{j}\right)(0 \leq j \leq N-2 m)$ we obtain $r_{j}=\sum_{\ell=0}^{m} \tilde{r}_{\ell} \cos \left(k \ell \pi x_{j}\right)$ for all $0 \leq j \leq N$. Hence, $\boldsymbol{r}=\sum_{\ell=0}^{m} \tilde{r}_{\ell} \boldsymbol{v}_{k \ell}$, i.e., $\boldsymbol{r} \in V_{k}^{N}$ holds.

Proof of Theorem 3. The operator $\mathcal{G}: V_{1}^{N} \rightarrow V_{1}^{N}$ is defined by $\mathcal{G}(\boldsymbol{t})=\boldsymbol{K} \boldsymbol{t}+\boldsymbol{M}(\boldsymbol{t}) \boldsymbol{t}-\boldsymbol{g}$. For $\boldsymbol{t} \in V_{k}^{N}$ we have $\boldsymbol{M}(\boldsymbol{t}) \boldsymbol{t} \in V_{k}^{N}$ due to Lemma 1 . Note that $\boldsymbol{g} \in V_{k}^{N}$ for all $k$ and $N$. We now consider the term $\boldsymbol{K} \boldsymbol{t}=\boldsymbol{V} \boldsymbol{K}_{S} \boldsymbol{V}^{-1} \boldsymbol{t}$ with $\boldsymbol{K}_{S}=\operatorname{diag}\left(d_{n}\right)_{0 \leq n \leq N}$. For $\boldsymbol{t} \in V_{k}^{N}$, with $m:=\frac{N}{k}$, we have $\boldsymbol{t}=\sum_{n=0}^{m} \alpha_{k n} \boldsymbol{v}_{k n}$. Thus

$$
\boldsymbol{V} \boldsymbol{K}_{S} \boldsymbol{V}^{-1} \boldsymbol{t}=\sum_{n=0}^{m} d_{k n} \alpha_{k n} \boldsymbol{v}_{k n}
$$

holds. This yields $\boldsymbol{K} \boldsymbol{t} \in V_{k}^{N}$ and completes the proof.

Proof of Theorem 4. Assume that $\boldsymbol{t}=\boldsymbol{t}^{*}$. Then $\sum_{n=0}^{m}\left(1-(-1)^{n}\right) \tilde{t}_{k n} \boldsymbol{v}_{k n}=0$ and thus $\tilde{t}_{k n}=0$ for all odd $n$. This yields $\boldsymbol{t}=\sum_{n=0}^{\left[\frac{1}{2} m\right]} \tilde{t}_{2 k n} \boldsymbol{v}_{2 k n}$, i.e., $\boldsymbol{t} \in V_{2 k}^{N}$, which contradicts the assumption $\boldsymbol{t} \notin V_{\ell}^{N}$ for $\ell>k$. Thus $\boldsymbol{t} \neq \boldsymbol{t}^{*}$. From

$$
t_{j}=\sum_{n=0}^{m} \tilde{t}_{k n} \cos \left(k n \pi x_{j}\right), \quad t_{j}^{*}=\sum_{n=0}^{m} \tilde{t}_{k n} \cos \left(k n \pi\left(x_{j}+\frac{1}{k}\right)\right)=\sum_{n=0}^{m} \tilde{t}_{k n} \cos \left(k n \pi x_{j+m}\right),
$$

for $0 \leq j \leq N$, it follows that

$$
t_{j}^{*}=t_{j+m} \quad \text { for } \quad 0 \leq j \leq N-m, \quad t_{j}^{*}=t_{2 N-m-j} \quad \text { for } \quad N-m \leq j \leq N .
$$

From

$$
\begin{aligned}
\mathcal{G}(\boldsymbol{t})_{j} & =\sum_{n=0}^{m} d_{k n} \tilde{t}_{k n} \cos \left(k n \pi x_{j}\right)+\alpha\left(t_{j}\right) t_{j}-\frac{1}{\Lambda} \\
\mathcal{G}\left(\boldsymbol{t}^{*}\right)_{j} & =\sum_{n=0}^{m} d_{k n} \tilde{t}_{k n} \cos \left(k n \pi x_{j+m}\right)+\alpha\left(t_{j}^{*}\right) t_{j}^{*}-\frac{1}{\Lambda},
\end{aligned}
$$

for $0 \leq j \leq N$, and (34) it follows that

$$
\mathcal{G}\left(\boldsymbol{t}^{*}\right)_{j}=\mathcal{G}(\boldsymbol{t})_{j+m} \quad \text { for } 0 \leq j \leq N-m, \quad \mathcal{G}\left(\boldsymbol{t}^{*}\right)_{j}=\mathcal{G}(\boldsymbol{t})_{2 N-m-j} \quad \text { for } \quad N-m \leq j \leq N .
$$

Since $\mathcal{G}(\boldsymbol{t})_{j}=0$ for all $0 \leq j \leq N$ we obtain $\mathcal{G}\left(\boldsymbol{t}^{*}\right)=0$. 